\title{
SOLVING PERIODIC SEMILINEAR STIFF PDES IN 1D, 2D AND 3D WITH EXPONENTIAL INTEGRATORS
}

\author{
HADRIEN MONTANELLI* AND NIALL BOOTLAND ${ }^{\dagger}$
}

\begin{abstract}
Dozens of exponential integration formulas have been proposed for the high-accuracy solution of stiff PDEs such as the Allen-Cahn, Korteweg-de Vries and Ginzburg-Landau equations. We report the results of extensive comparisons in MATLAB and Chebfun of such formulas in 1D, 2D and 3D, focusing on fourth and higher order methods, and periodic semilinear stiff PDEs with constant coefficients. Our conclusion is that it is hard to do much better than one of the simplest of these formulas, the ETDRK4 scheme of Cox and Matthews.
\end{abstract}

Key words. Stiff PDEs, exponential integrators, Fourier spectral methods, Chebfun

AMS subject classifications. 65L04, 65L05, 65M20, 65M70

1. Introduction. We are interested in computing smooth solutions of stiff PDEs of the form

$$
u_{t}=\mathcal{S}(u)=\mathcal{L} u+\mathcal{N}(u), \quad u(0, X)=u_{0}(X), \quad \text { periodic boundary conditions },
$$

where $u(t, X)$ is a function of time $t$ and space $X, \mathcal{L}$ is a linear differential operator with constant coefficients on a domain in one, two or three space dimensions and $\mathcal{N}$ is a nonlinear differential (or non-differential) operator of lower order with constant coefficients and on the same domain 1 In applications, PDEs of this kind typically arise when two or more different physical processes are combined, and many PDEs of interest in science and engineering take this form. For example, the Korteweg-de Vries equation $u_{t}=-u_{x x x}-u u_{x}$, the starting point of the study of nonlinear waves and solitons, couples third-order linear dispersion with first-order convection, and the Allen-Cahn equation $u_{t}=\epsilon u_{x x}+u-u^{3}$ couples second-order linear diffusion with a nondifferentiated cubic reaction term. Often a system of equations rather than a single scalar equation is involved, for example in the Gray-Scott and Schnakenberg equations, which involve two components coupled together. (The importance of coupling of nonequal diffusion constants in science was made famous by Alan Turing in the most highly-cited of all his papers 58.) Fourth-order terms also arise, for example in the Cahn-Hilliard equation, whose solutions describe structures of alloys, and in the Kuramoto-Sivashinsky equation, related to combustion problems among others, whose solutions are chaotic. Other examples of stiff PDEs include the Ginzburg-Landau, nonlinear Schrödinger (NLS) and Swift-Hohenberg equations. Figure 1.1 shows six examples of solutions of such PDEs.

Solving all these PDEs by generic numerical methods can be highly challenging. One of the main issues is stiffness, characterised by the need for an explicit method to use small time-steps, much smaller than the condition required by accuracy. When too many steps are required, this can result in an infeasibly long computation. There are other important issues such as the numerical conservation of various properties (e.g., for the KdV and NLS equations) - we shall not discuss this here; see, e.g., [10.

${ }^{*}$ Oxford University Mathematical Institute, Oxford OX2 6GG, UK. Supported by the European Research Council under the European Union's Seventh Framework Programme (FP7/2007-2013)/ERC grant agreement no. 291068. The views expressed in this article are not those of the ERC or the European Commission, and the European Union is not liable for any use that may be made of the information contained here.

†Oxford University Mathematical Institute, Oxford OX2 6GG, UK. This publication was based on work supported in part by award no. KUK-C1-013-04, made by King Abdullah University of Science and Technology (KAUST).

${ }^{1} X$ denotes a space variable in $1 \mathrm{D}, 2 \mathrm{D}$ or $3 \mathrm{D}$. Throughout this paper, we will use the variables $x$ in $1 \mathrm{D},(x, y)$ in $2 \mathrm{D}$ and $(x, y, z)$ in $3 \mathrm{D}$. The domain will be an interval in $1 \mathrm{D}$, a square in $2 \mathrm{D}$ and a cube in 3D. 
1D Allen-Cahn

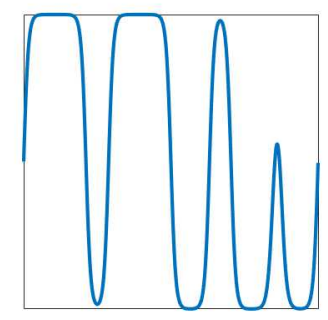

2D Ginzburg-Landau

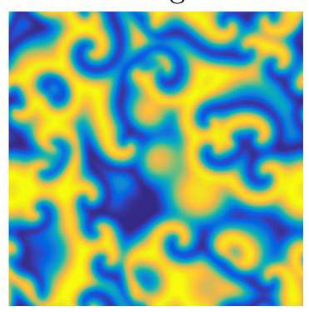

$1 \mathrm{D} \mathrm{KdV}$

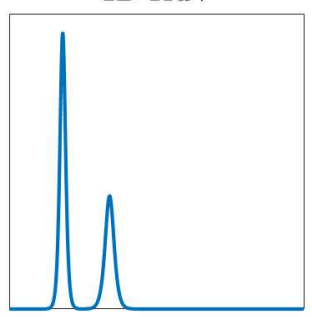

2D Schnakenberg

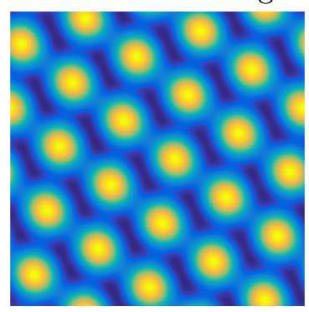

1D NLS

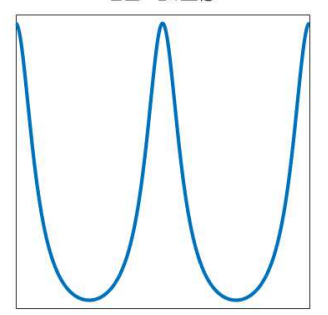

2D Swift-Hohenberg

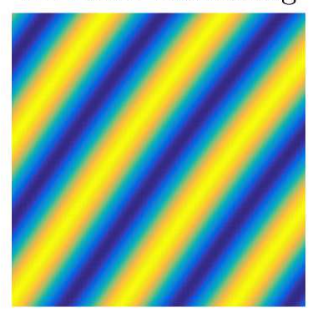

Fig. 1.1: First row (left to right): metastable solution of the Allen-Cahn equation (3.1), two-soliton solution of the KdV equation (3.7) and breather solution of the NLS equation (3.16). Second row (left to right): frozen state solution of the Ginzburg-Landau equation (3.24), spot solution of the Schnakenberg equations (3.27) and convection rolls of the Swift-Hohenberg equations (3.30).

This paper describes and compares specialized methods that take advantage of two special features of (1.1). The first one is the periodic boundary conditions. This allows us to discretize the spatial component of (1.1) with a Fourier spectral method on $N$ points; equation (1.1) becomes a system of $N$ ODEs,

$$
\hat{u}^{\prime}=\mathbf{S}(\hat{u})=\mathbf{L} \hat{u}+\mathbf{N}(\hat{u}), \quad \hat{u}(0)=\hat{u}_{0},
$$

where $\hat{u}(t)$ is the vector of $N$ Fourier coefficients of the trigonometric interpolant of $u(t, X)$ at time $t$, and $\mathbf{L}$ (a $N \times N$ matrix) and $\mathbf{N}$ are the discretized versions of $\mathcal{L}$ and $\mathcal{N}$ in Fourier space. For example, in $1 \mathrm{D}$ on $[0,2 \pi]$ with $\mathcal{L} u=u_{x x}$ and an even number $N$ of equispaced grid points $\left\{x_{j}=2 \pi j / N\right\}_{j=0}^{N-1}$, we look for a solution $u(t, x)$ of the form 2

$$
u(t, x) \approx \sum_{k=-N / 2}^{N / 2} \hat{u}_{k}(t) e^{i k x}
$$

\footnotetext{
${ }^{2}$ The prime on the summation sign in (1.3) signifies that the terms $k= \pm N / 2$ are halved.
} 
with Fourier coefficients

$$
\hat{u}_{k}(t)=\frac{1}{N} \sum_{j=0}^{N-1} u\left(t, x_{j}\right) e^{-i k x_{j}}, \quad-\frac{N}{2} \leq k \leq \frac{N}{2}-1, \quad \hat{u}_{N / 2}(t)=\hat{u}_{-N / 2}(t) .
$$

Since FFT codes only store $N$ coefficients, the vector $\hat{u}(t)$ is defined as

$$
\hat{u}(t)=\left(\frac{\hat{u}_{-N / 2}}{2}+\frac{\hat{u}_{N / 2}}{2}, \hat{u}_{-N / 2+1}(t), \ldots, \hat{u}_{N / 2-1}(t)\right)^{T} .
$$

For this PDE, $\mathbf{L}=\mathbf{D}_{N}^{(2)}$ is the (diagonal) second-order Fourier differentiation matrix with entries $-k^{2},-N / 2 \leq k \leq N / 2-1$. In Section 3, we will also consider the first-, third-, and fourth-order Fourier differentiation matrices $\mathbf{D}_{N}, \mathbf{D}_{N}^{(3)}$, and $\mathbf{D}_{N}^{(4)}$; see [55] for more details about Fourier spectral methods and [60] for a review of trigonometric interpolation techniques. (Note that stiffness is related to $\mathbf{L}$ having large eigenvalues since stability of spectral methods for time-dependent PDEs requires that the eigenvalues of $\mathbf{L}$, scaled by the time-step, lie in the stability region of the timestepping formula [55, Chapter 10].)

The second special feature of (1.1) is that it is semilinear, i.e., the higher-order terms of the equation are linear. Exponential integrators are a class of numerical methods for systems of ODEs that are aimed at taking advantage of this. The linear part $\mathbf{L}$, responsible for the stiffness, is integrated exactly using the matrix exponential while a numerical scheme is applied to $\mathbf{N}$.

According to the 2005 review of Minchev and Wright [37, the first exponential integrators were constructed by Certaine in 1960 [11] and Pope in 1963 [41]. Subsequently, however, Hochbruck and Ostermann [22] noted, in a comprehensive theoretical review of these schemes, that Hersch [19] had previously considered exponential integrators in 1958 in an effort to find schemes that are exact for linear problems with constant coefficients. The first use of the term exponential integrator was by Hochbruck, Lubich and Selhofer [20] in a seminal paper of 1998. The extensive use of these formulas for solving stiff PDEs seems to have been initiated by the papers by Cox and Matthews [12] and Kassam and Trefethen [26]. A striking unpublished paper by Kassam [25] shows how effective such methods can be also for PDEs in 2D and 3D. A software package for such computations called EXPINT was produced by Berland, Skaflestad and Wright [4].

One of the simplest exponential integrators, commonly known as the Exponential Time Differencing (ETD) Euler method, is given by 3

$$
\hat{u}^{n+1}=e^{h \mathbf{L}} \hat{u}^{n}+h \varphi_{1}(h \mathbf{L}) \mathbf{N}\left(\hat{u}^{n}\right),
$$

where $h=t_{n+1}-t_{n}$ is the time-step and

$$
\varphi_{1}(z)=\frac{e^{z}-1}{z}
$$

As Minchev and Wright [37] point out, this method has been rediscovered from many different viewpoints and has been known by several other names. It can be derived by considering the linearized version of (1.2) on $\left[t_{n}, t_{n+1}\right]$,

$$
\hat{u}^{\prime}=\mathbf{S}\left(\hat{u}^{n}\right)+\mathbf{S}_{\hat{u}}\left(\hat{u}^{n}\right)\left(\hat{u}-\hat{u}^{n}\right), \quad \hat{u}\left(t_{n}\right)=\hat{u}^{n},
$$

\footnotetext{
${ }^{3}$ Throughout this paper, when introducing an exponential integrator such as (1.6), $\hat{u}^{n}$ will mean $\hat{u}(t)$ at $t=t_{n}$.
} 
with exact solution at $t_{n+1}=t_{n}+h$,

$$
\hat{u}^{n+1}=\hat{u}^{n}+h \varphi_{1}\left(h \mathbf{S}_{\hat{u}}\left(\hat{u}^{n}\right)\right) \mathbf{S}\left(\hat{u}^{n}\right) .
$$

Approximating $\mathbf{S}_{\hat{u}}\left(\hat{u}^{n}\right)$ by $\mathbf{L}$ in (1.9) leads to (1.6). Note that (1.9) defines a time-stepping scheme too, known as the exponential Euler method. The problem with (1.9) is that the exact Jacobian $\mathbf{S}_{\hat{u}}$, and its value under the exponential-like function $\varphi_{1}$, need to be computed at each time-step. This would involve a high computational cost, so typically one either does not compute $\varphi_{1}$ but rather an approximation, such as a Padé approximation, or else one uses an approximation to the Jacobian as opposed to the exact Jacobian. The former approach contains the Rosenbrock methods [17, 18, 24, 35, 44, 59] and the Exponential Propagation Iterative methods of RungeKutta type (EPIRK) [42, 43, 51, 52, 54]. The latter approach is what we shall consider in this paper.

As we just described, exponential integrators are characterised by the use of exponential and related functions of the matrix $\mathbf{L}$. Standard methods for computing the matrix exponential in the context of exponential integrators include the scaling and squaring method [1, the CarathéodoryFejér method [45] and Krylov subspace methods [51]. There is recent work that shows that exponential integrators together with Krylov methods are competitive, for instance see Tokman and Loffeld [33, 53]. In our case we consider periodic problems with constant coefficients so the matrices are diagonal and the matrix exponential is trivial.

We compare in this paper 30 exponential integrators of fourth and higher order on 11 model problems in 1D, 2D and 3D, using MATLAB R2015b and Chebfun v5.5 [13. Comparisons with other types of time-stepping schemes are out of the scope of the article; see, e.g., [16, 25, 27, 28, 33. Let us emphasize that we are interested in determining if one of the high order integrators outperforms the others on a large class of problems. For a particular problem, it might be possible to design a very specific scheme, of possibly lower order than four, which performs extremely well. For example, Cano and Gonzáles-Pachón have recently shown that the low-order Lawson methods, combined with orthogonal projections onto some invariants, can be very competitive for the nonlinear Schrödinger equation [9, 10. Let us also emphasize that since we only consider periodic problems, we do not expect to see any order reduction in the convergence of the exponential integrators, as already observed in, e.g., [26. For different types of boundary conditions (e.g., homogeneous Dirichlet conditions), certain schemes (e.g., Lawson methods) do not satisfy the socalled stiff order conditions [22] - which guarantee a certain order of convergence independently of the considered problem - and can therefore exhibit a strong order reduction in practice.

The paper is structured as follows. We present the 30 exponential integrators in Section 2 and the 11 model problems in Section 3. The numerical results are presented in Section 4 and show that it is hard to do much better than one of the simplest of these formulas, the ETDRK4 scheme of Cox and Matthews [12].

\section{Thirty exponential integrators.}

2.1. Exponential general linear methods. We consider exponential integrators, based on the approximation of the Jacobian of (1.9), that belong to the large class of exponential general linear methods, first introduced by Minchev and Wright in 2005 [37. This class contains, in particular, the ETD Runge-Kutta (one-step), ETD Adams-Bashforth (multistep), Lawson and exponential predictor-corrector methods. For given starting values $\hat{u}^{0}, \hat{u}^{1}, \ldots, \hat{u}^{q-1}$ at times $t=0, h, \ldots,(q-1) h$, 
Table 2.1: Butcher tableau of an exponential integrator with $q$ steps and s stages.

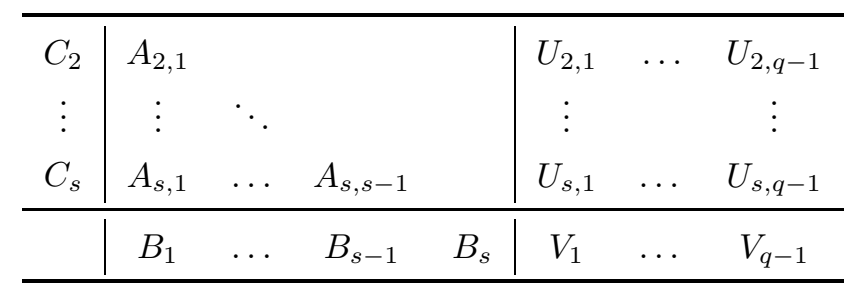

the numerical approximation $\hat{u}^{n+1}$ at time $t_{n+1}=(n+1) h, n+1 \geq q$, is given by the formula

$$
\hat{u}^{n+1}=e^{h \mathbf{L}} \hat{u}^{n}+h \sum_{i=1}^{s} B_{i}(h \mathbf{L}) \mathbf{N}\left(\hat{v}^{i}\right)+h \sum_{i=1}^{q-1} V_{i}(h \mathbf{L}) \mathbf{N}\left(\hat{u}^{n-i}\right),
$$

with $q$ steps $\hat{u}^{n-i}$ and $s$ stages $\hat{v}^{i}$, with $\hat{v}^{1}=\hat{u}^{n}$ and

$$
\hat{v}^{i}=e^{C_{i} h \mathbf{L}} \hat{u}^{n}+h \sum_{j=1}^{i-1} A_{i, j}(h \mathbf{L}) \mathbf{N}\left(\hat{v}^{j}\right)+h \sum_{j=1}^{q-1} U_{i, j}(h \mathbf{L}) \mathbf{N}\left(\hat{u}^{n-j}\right), \quad 2 \leq i \leq s .
$$

Each scheme is characterised by its coefficients $A, B, C, U$, and $V$, which can be conveniently listed in a Butcher tableau, as in Table 2.1. Note that these coefficients (except $C$ ) depend on $\mathbf{L}$ - for instance, (1.6) uses one stage and one step, and its only non-zero coefficient is $B_{1}=\varphi_{1}(h \mathbf{L})$. Note that, in practice, the nonlinear evaluations $\mathbf{N}\left(\hat{v}^{i}\right)$ and $\mathbf{N}\left(\hat{u}^{n-i}\right)$ are carried out in value space, e.g., $\mathbf{N}\left(\hat{v}^{i}\right)$ means $\mathbf{F}\left(\mathbf{N}\left(\mathbf{F}^{-1} \hat{v}^{i}\right)\right)$, with discrete Fourier transform $\mathbf{F}$. Methods of the form (2.1) -(2.2) not only include purely one-step methods $(q=1, s \geq 1)$ and purely multistep methods $(q \geq 1$, $s=1$ ), but also combinations of both.

The coefficients satisfy the following summation properties 4

$$
\begin{aligned}
& B_{1}=\varphi_{1}(h \mathbf{L})-\sum_{i=2}^{s} B_{i}(h \mathbf{L})-\sum_{i=1}^{q-1} V_{i}(h \mathbf{L}), \\
& A_{i, 1}=\psi_{1, i}(h \mathbf{L})-\sum_{j=2}^{i-1} A_{i, j}(h \mathbf{L})-\sum_{j=1}^{q-1} U_{i, j}(h \mathbf{L}), \quad 2 \leq i \leq s,
\end{aligned}
$$

where the $\varphi$ - and $\psi$-functions are exponential and related functions that we shall define in the next subsection. As a consequence, it is notationally convenient to incorporate this condition by filling the corresponding entries of the Butcher tableau with a dot on the understanding that these method coefficients are given by (2.3). Note that exponential integrators of the form (2.1) - (2.2) do not include the EMAM4 scheme of Calvo and Palencia [8]. It has been shown in [6] that it often suffers from stability problems.

\footnotetext{
${ }^{4}$ There are two exceptions: the coefficients of the Lawson4 and ABLawson4 schemes do not satisfy the summation properties (2.3).
} 
Let us finish this section with a few words about the computational cost per time-step. Since the matrices in (2.1)- 2.2) are diagonal, the matrix-vector products cost only $\mathcal{O}(N)$ operations. The dominant cost per time-step is then the cost of an FFT, i.e., $\mathcal{O}(N \log N)$ operations. For exponential integrators of the form (2.1) $-(2.2)$, the total cost to compute $\hat{u}^{n+1}$ is therefore $\mathcal{O}(2 s N \log N)$. As a consequence, purely multistep methods have a low computational cost per time-step.

2.2. Evaluating the $\varphi$-functions. The coefficients $A, B, C, U$, and $V$ involve the $\varphi$ and $\psi$-functions applied to $\mathbf{L}$. Because $\mathbf{L}$ is diagonal, $\varphi(\mathbf{L})$ and $\psi(\mathbf{L})$ reduce to $\varphi$ and $\psi$ applied to the diagonal elements $\lambda$ of $\mathbf{L}$, so all we have to be able to is to compute $\varphi(\lambda)$ and $\psi(\lambda)$ for $\lambda \in \mathbb{C}$. The $\varphi$-functions are defined by the recurrence relation,

$$
\varphi_{l+1}(z)=\frac{\varphi_{l}(z)-1 / l !}{z}, \quad l \geq 1
$$

with $\varphi_{0}(z)=e^{z}$. After $\varphi_{0}$, the first few $\varphi$-functions are (1.7) and

$$
\varphi_{2}(z)=\frac{e^{z}-z-1}{z^{2}}, \quad \varphi_{3}(z)=\frac{e^{z}-\frac{z^{2}}{2}-z-1}{z^{3}}
$$

while the $\psi$-functions are defined via the $\varphi$-functions and the coefficients $C$,

$$
\psi_{l, m}(z)=C_{m}^{l} \varphi_{l}\left(C_{m} z\right), \quad l \geq 0, \quad 1 \leq m \leq s
$$

Equations (2.4) and (2.6) can be implemented recursively, but the accurate evaluation of $\varphi$ and $\psi$ is not straightforward because it can suffer from cancellation error. Following the idea of Kassam and Trefethen in [26], to compute the functions at some $\lambda \in \mathbb{C}$, we use Cauchy's integral formula

$$
\varphi(\lambda)=\frac{1}{2 \pi i} \oint_{\Gamma} \frac{\varphi(z)}{z-\lambda} d z
$$

which can be approximated with exponential accuracy by the trapezoidal rule [56],

$$
\varphi(\lambda) \approx \frac{1}{M} \sum_{k=1}^{M} \varphi\left(\lambda+e^{2 \pi i(k-0.5) / M}\right)
$$

taking $\Gamma$ to be the circle of radius 1 centred at $\lambda$, oriented counter-clockwise, discretized with $M$ equally spaced points. Note that the $\varphi$-functions satisfy $\varphi(\bar{z})=\bar{\varphi}(z)$ for all $z \in \mathbb{C}$. As a consequence, when $\lambda$ is on the real axis, we can take $\Gamma$ to be the upper half of the circle of radius 1 centred at $\lambda$ and take the real part of the result, i.e.,

$$
\varphi(\lambda)=\frac{1}{\pi} \mathcal{R}\left(\int_{0}^{\pi} \varphi\left(\lambda+e^{i \theta}\right) d \theta\right)
$$

which can be discretized by

$$
\varphi(\lambda) \approx \frac{1}{M} \mathcal{R}\left(\sum_{k=1}^{M} \varphi\left(\lambda+e^{\pi i(k-0.5) / M}\right)\right) .
$$


If this symmetry is not explicitly used in the computation of the $\varphi$-functions when $\lambda$ is real, rounding errors appear that lead to numerical instability. Note that the evaluation of the $\varphi$-functions using (2.8) or (2.10) requires $\mathcal{O}(M)$ operations per $\lambda$.

Let us emphasize that we can use circles of radius 1 around each eigenvalue $\lambda$ of $\mathbf{L}$ because $\mathbf{L}$ is diagonal. When the matrix is not diagonal, one has to use a single contour that encloses all the eigenvalues, and the best possible contour depends on the problem. For example, for parabolic problems (also called diffusive problems), all the eigenvalues are on the real negative axis and the best contour is a Hankel contour [45, 57].

Using contour integrals is not the only possible remedy for cancellation error. When Pope introduced (1.6), he suggested the use of Taylor series for small $\lambda$ and the direct formula for large $\lambda$. The problem with this approach is that, for some intermediate values, neither method gives full precision, as noted by Cox and Matthews [12] and Kassam and Trefethen [26]. Another approach is to use Padé approximations, combined with a scaling and squaring technique [5]. This method is also effective, but the contour integral method is particularly appealing because of its greater generality for dealing with arbitrary functions.

2.3. Introducing the 30 exponential integrators. Table 2.2 lists the exponential integrators considered in this paper. Their Butcher tableaux can be found in the Ph.D. thesis of the first author [38].

ETD Adams-Bashforth. The first category of exponential integrators is the ETD AdamsBashforth schemes of order four to six. These are ETD (purely) multistep methods, which reduce to Adams-Bashforth schemes when $\mathbf{L}=0$, and go back to Nørsett in 1969 [39]. Since $s=1$, (2.1) - (2.2) takes the simpler form

$$
\hat{u}^{n+1}=e^{h \mathbf{L}} \hat{u}^{n}+h B_{1}(h \mathbf{L}) \mathbf{N}\left(\hat{u}^{n}\right)+h \sum_{i=1}^{q-1} V_{i}(h \mathbf{L}) \mathbf{N}\left(\hat{u}^{n-i}\right),
$$

i.e., the only non-zero coefficients are $B_{1}$ and those in $V$. Note that since these schemes are purely multistep, (2.11) only requires two FFTs per time-step. We label these methods as ABNørsettq, where $4 \leq q \leq 6$ is the order and also the number of steps. For more details on the derivation of ABNørsett methods see Minchev and Wright [37], who also show a connection between these schemes and the IMEX schemes of Ascher, Ruuth and Wetton [3. One can also derive methods based on Adams-Moulton methods, known as AMNørsett $q$ methods. These are implicit but can be used within predictor-corrector pairs, as we will see when introducing exponential predictorcorrector schemes (the last category in the table). A comprehensive look at both the AdamsBashforth and Adams-Moulton exponential integrators can be found in the paper by Hochbruck and Ostermann [23].

ETD Runge-Kutta. The second category is the ETD Runge-Kutta schemes of order four to five. These are (purely) one-step methods and go back to Friedli in 1978 [15] and Strehmel-Weiner in 1982 [49. More recently, inspired by Cox and Matthews' ETDRK4 scheme [12], Minchev [36] in 2004 and Krogstad [30] and Hochbruck and Ostermann 21] in 2005 derived ETD Runge-Kutta schemes of order four. Luan and Ostermann proposed a scheme of order five (EXPRK5S8) in 2014 [34]. Overviews of ETD Runge-Kutta methods and some of their history can be found in the reviews of Hochbruck and Ostermann [22] and Minchev and Wright [37, where connections are described between ETD Runge-Kutta, generalised Runge-Kutta and semi-implicit methods. Since 
Table 2.2: A reference table for the exponential integrators considered in this paper. Since there is no order reduction for periodic (diagonal) problems, the stiff convergence order is the same at the non-stiff convergence order. Note that some methods do not appear explicitly in the references listed but can be derived using order conditions or recurrence formulas found there; these cases are marked by asterisks. The Butcher tableaux can be found in the Ph.D. thesis of the first author [38].

\begin{tabular}{|c|c|c|c|c|c|}
\hline Method & Type & Order & Stages $s$ & Steps $q$ & Ref. \\
\hline ABNørsett4 & ETD Adams-Bashforth & 4 & 1 & 4 & 39 , \\
\hline ABNørsett5 & ETD Adams-Bashforth & 5 & 1 & 5 & 39 . \\
\hline ABNørsett6 & ETD Adams-Bashforth & 6 & 1 & 6 & 39 . \\
\hline ETDRK4 & ETD Runge-Kutta & 4 & 4 & 1 & 12 \\
\hline Friedli & ETD Runge--Kutta & 4 & 4 & 1 & 15 \\
\hline Krogstad & ETD Runge-Kutta & 4 & 4 & 1 & 30 \\
\hline Minchev & ETD Runge-Kutta & 4 & 4 & 1 & 36 \\
\hline Strehmel-Weiner & ETD Runge-Kutta & 4 & 4 & 1 & 49 \\
\hline Hochbruck-Ostermann & ETD Runge-Kutta & 4 & 5 & 1 & 21 \\
\hline EXPRK5S8 & ETD Runge-Kutta & 5 & 8 & 1 & 34 \\
\hline ABLawson4 & Lawson & 4 & 1 & 4 & 32 \\
\hline Lawson4 & Lawson & 4 & 4 & 1 & 32 \\
\hline GenLawson41 & Gen. Lawson & 4 & 4 & 1 & 30 \\
\hline GenLawson 42 & Gen. Lawson & 4 & 4 & 2 & 30 \\
\hline GenLawson43 & Gen. Lawson & 4 & 4 & 3 & 30 \\
\hline GenLawson 44 & Gen. Lawson & 5 & 4 & 4 & 30 \\
\hline GenLawson45 & Gen. Lawson & 6 & 4 & 5 & 30 \\
\hline ModGenLawson41 & Mod. Gen. Lawson & 4 & 4 & 1 & 40 ] \\
\hline ModGenLawson 42 & Mod. Gen. Lawson & 4 & 4 & 2 & [40] $]^{*}$ \\
\hline ModGenLawson43 & Mod. Gen. Lawson & 4 & 4 & 3 & 40 . \\
\hline ModGenLawson44 & Mod. Gen. Lawson & 5 & 4 & 4 & $40]^{*}$ \\
\hline ModGenLawson45 & Mod. Gen. Lawson & 6 & 4 & 5 & $40]^{*}$ \\
\hline $\mathrm{PEC} 423$ & Exp. Predictor-Corrector & 4 & 2 & 3 & $40{ }^{*}$ \\
\hline PECEC433 & Exp. Predictor-Corrector & 4 & 3 & 3 & $40{ }^{*}$ \\
\hline PEC524 & Exp. Predictor-Corrector & 5 & 2 & 4 & 40 ] \\
\hline PECEC534 & Exp. Predictor-Corrector & 5 & 3 & 4 & [40] $]^{*}$ \\
\hline PEC625 & Exp. Predictor-Corrector & 6 & 2 & 5 & $40{ }^{*}$ \\
\hline PECEC635 & Exp. Predictor-Corrector & 6 & 3 & 5 & $40]^{*}$ \\
\hline PEC726 & Exp. Predictor-Corrector & 7 & 2 & 6 & [40] $]^{*}$ \\
\hline PECEC736 & Exp. Predictor-Corrector & 7 & 3 & 6 & $40{ }^{*}$ \\
\hline
\end{tabular}


Table 2.3: Butcher tableau for ETDRK4. Note the dots in the first column, which indicate that these coefficients are computed using (2.3). These coefficients are (from top to bottom): $A_{2,1}=\psi_{1,2}$, $A_{3,1}=0, A_{4,1}=\varphi_{1}-2 \psi_{1,2}$ and $B_{1}=\varphi_{1}-3 \varphi_{2}+4 \varphi_{3}$.

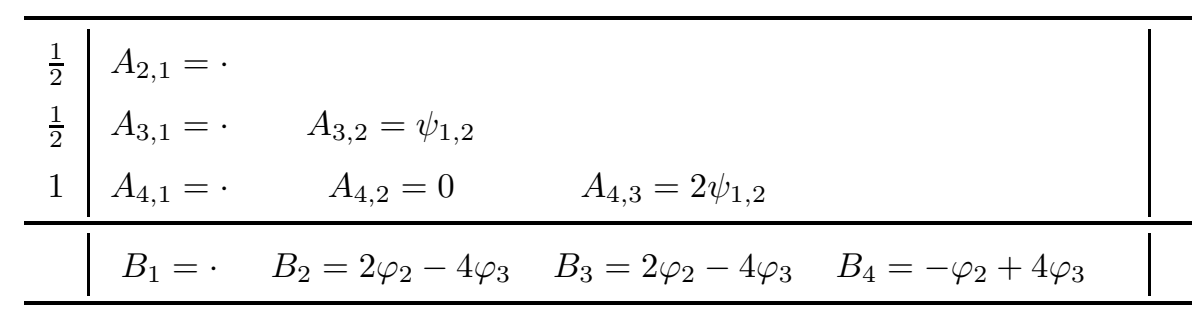

$q=1,(2.1)-(2.2)$ reduces to

$$
\begin{aligned}
& \hat{u}^{n+1}=e^{h \mathbf{L}} \hat{u}^{n}+h \sum_{i=1}^{s} B_{i}(h \mathbf{L}) \mathbf{N}\left(\hat{v}^{i}\right), \\
& \hat{v}^{1}=\hat{u}^{n}, \quad \hat{v}^{i}=e^{C_{i} h \mathbf{L}} \hat{u}^{n}+h \sum_{j=1}^{i-1} A_{i, j}(h \mathbf{L}) \mathbf{N}\left(\hat{v}^{j}\right), \quad 2 \leq i \leq s .
\end{aligned}
$$

The only non-zero coefficients are those in $A, B$ and $C$. The coefficients for the ETDRK4 scheme can be found in Table 2.3 and correspond to the following formula:

$$
\begin{aligned}
& \hat{v}^{1}=\hat{u}^{n}, \\
& \hat{v}^{2}=e^{\mathbf{L} h / 2} \hat{u}^{n}+(h / 2) \varphi_{1}(\mathbf{L} h / 2) \mathbf{N}\left(\hat{v}^{1}\right), \\
& \hat{v}^{3}=e^{\mathbf{L} h / 2} \hat{u}^{n}+(h / 2) \varphi_{1}(\mathbf{L} h / 2) \mathbf{N}\left(\hat{v}^{2}\right), \\
& \hat{v}^{4}=e^{\mathbf{L} h / 2} \hat{v}^{2}+(h / 2) \varphi_{1}(\mathbf{L} h / 2)\left[2 \mathbf{N}\left(\hat{v}^{3}\right)-\mathbf{N}\left(\hat{v}^{1}\right)\right], \\
& \hat{u}^{n+1}=e^{\mathbf{L} h} \hat{u}^{n}+h B_{1} \mathbf{N}\left(\hat{v}^{1}\right)+h B_{2}\left[\mathbf{N}\left(\hat{v}^{2}\right)+\mathbf{N}\left(\hat{v}^{3}\right)\right]+h B_{4} \mathbf{N}\left(\hat{v}^{4}\right),
\end{aligned}
$$

where $B_{1}=B_{1}(h \mathbf{L}), \ldots, B_{4}=B_{4}(h \mathbf{L})$.

Lawson. The third category is the Lawson methods. First developed by Lawson in 1967 [32], and often known as integrating factor (IF) methods, the motivation behind Lawson methods is to use a change of variable in (1.2) to get rid of the stiff linear part, and then apply a numerical solver to the transformed equation. The Lawson transformation consists of the change of variables $\hat{v}(t)=e^{-\mathbf{L} t} \hat{u}(t)$. If we differentiate this and substitute into (1.2), the transformed equation is

$$
\hat{v}^{\prime}=e^{-\mathbf{L} t} \mathbf{N}\left(e^{\mathbf{L} t} \hat{v}\right), \quad \hat{v}(0)=\hat{u}_{0} .
$$

The linear term is gone, and the transformed equation (2.14), while no longer stiff, now has rapidly varying coefficients. Once we have decided on a scheme to solve (2.14), we can transform back to $\hat{u}$. Lawson, in his 1967 paper, used the classical fourth order Runge-Kutta scheme on the transformed 
equation (2.14); we call this method Lawson4. Using the classical fourth order Adams-Bashforth scheme gives the ABLawson4 method. Ehle and Lawson observed in [14 that Runge-Kutta based Lawson methods only work well when the problem is moderately stiff. Another problem with Lawson methods, as indicated by Krogstad [30], is that they do not preserve fixed points of the differential equation.

Generalised Lawson. Krogstad worked around these problems to derive generalised Lawson methods, also called generalised integrating factor (GIF) methods, the fourth category in the table. These are based on the transform

$$
\hat{v}(t)=e^{-\mathbf{L} t} \hat{u}(t)-e^{-\mathbf{L} t} \sum_{l=1}^{q} t^{l} \varphi_{l}(t \mathbf{L}) p_{l-1},
$$

where the $p_{l}$ are the coefficients, in a (scaled) monomial basis, of the polynomial $P(t)$ of degree $q-1$ that interpolates the values $\left\{N\left(\hat{u}^{n-l}\right)\right\}_{l=1}^{q}$ at the points $\left\{t_{n-l}\right\}_{l=1}^{q}$; see [22, 37] for details. Differentiating this and substituting into (1.2) leads to the transformed equation

$$
\hat{v}^{\prime}=e^{-\mathbf{L} t}\left(\mathbf{N}\left(e^{\mathbf{L} t} \hat{v}+\sum_{l=1}^{q} t^{l} \varphi_{l}(t \mathbf{L}) p_{l-1}\right)-P(t)\right), \quad \hat{v}(0)=\hat{u}_{0} .
$$

Note that (2.14) is the special case of (2.16) with $P(t)=0$. The idea of Krogstad is to apply, for various values of $q$, the classical fourth order Runge-Kutta scheme on (2.16), and then transform back to $\hat{u}$. It leads to methods with four stages and $q$ steps, called the GenLawson $4 q$ methods.

Modified generalised Lawson. As we increase $q$ in the generalised Lawson methods we incorporate more of the nonlinearity and the methods have improved accuracy. However, this in part comes at the cost of stability, especially for dispersive problems, as was demonstrated by Krogstad [30]. A modification, based on satisfying order conditions, given by Ostermann, Thalhammer and Wright [40], significantly improves stability. The modification is given by the requirement that

$$
\sum_{i=1}^{4} B_{i}(h \mathbf{L}) \frac{c_{i}^{j}}{j !}+\sum_{i=1}^{q-1} V_{i}(h \mathbf{L}) \frac{(-1)^{j}}{j !}=\varphi_{j+1}(h \mathbf{L}), \quad 0 \leq j \leq q-1,
$$

where, as before, $q-1$ is the degree of the polynomial approximation. These are the modified generalised Lawson methods, labelled as ModGenLawson4q.

Exponential predictor-corrector. Just as with the standard Adams-Bashforth and AdamsMoulton multistep methods, the exponential versions can be used in predictor-corrector pairs. These are the exponential predictor-corrector methods, the last category in the table. For instance, using $\mathrm{ABN} \varnothing \mathrm{rsett} 3$ for a predictor step and $\mathrm{AMN} \varnothing \mathrm{rsett} 4$ for the corrector step yields the fourth order method called PEC423 in the MATLAB package EXPINT [4]. (PEC stands for predict-evaluatecorrect, four is the order, two is the number of stages and three is the number of steps.) One can evaluate and correct again, that is, use the corrector twice. The name PECEC433 is given in EXPINT for the fourth order method that uses ABNørsett3 for a predictor step and AMNørsett4 for two corrector steps.

3. Eleven model problems. In this section we describe the PDEs used in the comparisons of Section 4, including the initial conditions, the domains and the time intervals. There are five PDEs in 1D and three PDEs considered in both 2D and 3D; see Table 3.1 . 
Table 3.1: The model problems we consider in this paper. The linear operator of a diffusive PDE has real eigenvalues while it has purely imaginary eigenvalues for dispersive PDEs. Note that we take $A=0$ for the Ginzburg-Landau equation (3.24). For $A \neq 0$, the linear part would have complex eigenvalues.

\begin{tabular}{ccc}
\hline PDE & Dimension & Stiff linear Part \\
\hline Allen-Cahn & 1D & second-order diffusive \\
Cahn-Hilliard & 1D & fourth-order diffusive \\
Korteweg-de Vries & 1D & third-order dispersive \\
Kuramoto-Sivashinsky & 1D & fourth-order diffusive \\
nonlinear Schrödinger & 1D & second-order dispersive \\
Ginzburg-Landau & 2D \& 3D & second-order diffusive \\
Schnakenberg & 2D \& 3D & second-order diffusive \\
Swift-Hohenberg & 2D \& 3D & fourth-order diffusive \\
\hline
\end{tabular}

\subsection{Model problems in 1D.}

Allen-Cahn. The Allen-Cahn equation, derived by Allen and Cahn in the 1970s, is a reactiondiffusion equation which describes the process of phase separation in iron alloys (see, e.g., [2]). It is given in one dimension as

$$
u_{t}=\epsilon u_{x x}+u-u^{3}
$$

with linear diffusion $\epsilon u_{x x}$ and a cubic reaction term $u-u^{3}$. The function $u$ is the order parameter, a correlation function related to the positions of the different components of the alloy. The Allen-Cahn equation exhibits stable equilibria at $u= \pm 1$ while $u=0$ is an unstable equilibrium. Solutions often display metastability where wells $u \approx-1$ compete with peaks $u \approx 1$, and structures remain almost unchanged for long periods of time before changing suddenly. This can be quantified: features with width $L$ persist for time scales on the order of $e^{L / \epsilon}$. In Fourier space with a grid of size $N$, (3.1) becomes

$$
\hat{u}^{\prime}=\epsilon \mathbf{D}_{N}^{(2)} \hat{u}+\hat{u}-\mathbf{F}\left(\left(\mathbf{F}^{-1} \hat{u}\right)^{3}\right) .
$$

We take $\epsilon=5 \times 10^{-2}$,

$$
u(0, x)=\frac{1}{3} \tanh (2 \sin (x))-e^{-23.5 \cdot(x-\pi / 2)^{2}}+e^{-27(x-4.2)^{2}}+e^{-38(x-5.4)^{2}},
$$

with $x \in[0,2 \pi]$ and solve up to $t=60$. This initial condition quickly converges to a set of wells $u \approx-1$ and peaks $u \approx 1$ (at around $t=4$ ) and eventually to a two-plateau solution (at around $t=500$ ). Figure 1.1 shows the solution at time $t=113$, when the peak on the far right is switching to $u \approx-1$. 
Cahn-Hilliard. The Cahn-Hilliard equation,

$$
u_{t}=\alpha\left(-u_{x x}-\gamma u_{x x x x}+\left(u^{3}\right)_{x x}\right),
$$

is a fourth order reaction-diffusion problem which Cahn and Hilliard proposed in 1958 as a model for the process of phase separation in binary alloys [7. It couples second-order destabilizing diffusion $-u_{x x}$ with fourth-order stabilizing diffusion $-u_{x x x x}$ and a differentiated cubic reaction term $\left(u^{3}\right)_{x x}$. The function $u$ is defined as $u=1-2 c_{A}$ where $0 \leq c_{A} \leq 1$ and $c_{B}=1-c_{A}$ denote the concentrations of the two components $A$ and $B$ of the alloy, that is, $u=-1$ means pure $A$ while $u=1$ means pure $B$. The Cahn-Hilliard equation also exhibits metastable solutions. When quenched below a critical temperature, alloys described by (3.4) become unstable in the sense that small metastable pockets of relatively pure $A$ and $B$ may soon appear, corresponding to wells $u=-1$ and peaks $u=1$. These pockets may coarsen into larger pockets at progressively larger times. In Fourier space, (3.4) becomes

$$
\hat{u}^{\prime}=\alpha\left(-\mathbf{D}_{N}^{(2)}-\gamma \mathbf{D}_{N}^{(4)}\right) \hat{u}+\alpha \mathbf{D}_{N}^{(2)} \mathbf{F}\left(\left(\mathbf{F}^{-1} \hat{u}\right)^{3}\right) .
$$

We take $\alpha=10^{-2}, \gamma=10^{-3}$,

$$
u(0, x)=\frac{1}{5} \sin (4 \pi x)^{5}-\frac{4}{5} \sin (\pi x), \quad x \in[-1,1],
$$

and solve up to $t=12$. This initial condition evolves to a four-plateau solution (two wells $u \approx-1$, two peaks $u \approx 1$ ) at around $t=12$ before switching to a two-plateau solution (one well, one peak) at around $t=70$.

Korteweg-de Vries. The KdV equation,

$$
u_{t}=-u_{x x x}-u u_{x}
$$

was derived by Korteweg and de Vries in 1895 to model the propagation of waves in shallow water [29]. It couples dispersion $-u_{x x x}$ with nonlinear convection $-u u_{x}$. Among the solutions of (3.7) are solitary waves or solitons. These are waves that maintain their shapes as they travel and are given by

$$
u(t, x)=3 c \operatorname{sech}^{2}\left(\frac{\sqrt{c}}{2}\left(x-x_{0}-c t\right)\right), \quad c>0 .
$$

Waves of the form (3.8) have amplitude $3 c$ and travel at constant speed $c$. This is contrast to solutions of linear wave equations $u_{t}=-c u_{x}$, which all travel at velocity $c$, regardless of their amplitudes. In Fourier space, (3.7) becomes

$$
\hat{u}^{\prime}=-\mathbf{D}_{N}^{(3)} \hat{u}-\frac{\mathbf{D}_{N}}{2} \mathbf{F}\left(\left(\mathbf{F}^{-1} \hat{u}\right)^{2}\right) .
$$

We take

$$
u(0, x)=3 A^{2} \operatorname{sech}^{2}\left(\frac{A}{2}(x+2)\right)+3 B^{2} \operatorname{sech}^{2}\left(\frac{B}{2}(x+1)\right), \quad x \in[-\pi, \pi],
$$

with $A=25$ and $B=16$, and solve up to $t=10^{-2}$. This is a superposition of two solitons with speed $A^{2}$ and $B^{2}$ initially centred at $x=-2$ and $x=-1$, respectively. The stronger wave $(A=25)$ 
catches up with the weaker one $(B=16)$ at around $t=10^{-3}$. Both waves remain unchanged after the interaction, the only nonlinear effect being a forward shift

$$
\frac{1}{A^{2}} \log \left(\frac{A^{2}+B^{2}}{A^{2}-B^{2}}\right)^{2}
$$

for the stronger wave and a backward shift

$$
-\frac{1}{B^{2}} \log \left(\frac{A^{2}+B^{2}}{A^{2}-B^{2}}\right)^{2}
$$

for the weaker one. The interaction ends at around $t=3.5 \times 10^{-3}$. Figure 1.1 shows the initial condition.

Kuramoto-Sivashinsky. The Kuramoto-Sivashinsky equation,

$$
u_{t}=-u_{x x}-u_{x x x x}-u u_{x},
$$

dates to the mid-1970s with the work of Kuramoto [31] and Sivashinsky [47]. It couples destabilizing $-u_{x x}$ and stabilizing $-u_{x x x x}$ diffusions with nonlinear convection $-u u_{x}$. The nonlinear term shifts energy created at low wavenumbers by the second-order term to high wavenumbers where the fourthorder term stabilises. The Kuramoto-Sivashinsky equation models various physical phenomena, from unstable drift waves in plasmas to thermal instabilities in laminar flame fronts. In the latter, the function $u$ represents the perturbation of the flame front surface. The solutions of (3.13) can demonstrate a wide range of spatio-temporal dynamics, including chaos. In Fourier space, (3.13) becomes

$$
\hat{u}^{\prime}=\left(-\mathbf{D}_{N}^{(2)}-\mathbf{D}_{N}^{(4)}\right) \hat{u}-\frac{\mathbf{D}_{N}}{2} \mathbf{F}\left(\left(\mathbf{F}^{-1} \hat{u}\right)^{2}\right)
$$

We take

$$
u(0, x)=\cos \left(\frac{x}{16}\right)\left(1+\sin \left(\frac{x}{16}\right)\right), \quad x \in[0,32 \pi],
$$

and solve up to $t=100$. This simple initial data progressively evolves into a much more complicated superposition of wavenumbers and, even though the solution looks quite complicated, a characteristic pattern emerges from $t \approx 50$.

Nonlinear Schrödinger. The (focusing) NLS equation,

$$
u_{t}=i u_{x x}+i|u|^{2} u
$$

models several physical phenomena, including the nonlinear propagation of light in optical fibres. A nonlinear variant of the Schrödinger equation, it couples dispersion $i u_{x x}$ with a nonlinear potential $i|u|^{2} u$. Note that the wave function $u$ is complex-valued. Among the solutions of (3.16) are breathers, given by

$$
u(t, x)=A\left(\frac{2 B^{2} \cosh (\theta)+2 i B \sqrt{2-B^{2}} \sinh (\theta)}{2 \cosh (\theta)-\sqrt{2} \sqrt{2-B^{2}} \cos (A B x)}-1\right) e^{i A^{2} t}
$$

with $\theta=A^{2} B \sqrt{2-B^{2}} t$ and $B \leq \sqrt{2}$. These are nonlinear waves in which energy concentrates in a localized and oscillatory fashion. In Fourier space, (3.16) becomes

$$
\hat{u}^{\prime}=i \mathbf{D}_{N}^{(2)} \hat{u}+i \mathbf{F}\left(\left|\mathbf{F}^{-1} \hat{u}\right|^{2} \mathbf{F}^{-1} \hat{u}\right) .
$$


We take

$$
u(0, x)=\frac{2 A B^{2}}{2-\sqrt{2} \sqrt{2-B^{2}} \cos (A B x)}-A, \quad x \in[-\pi, \pi],
$$

with $A=2$ and $B=1$, and solve up to $t=2$. This is a breather whose amplitude oscillates in time around $A=2$. Figure 1.1 shows the initial condition.

3.2. Model problems in $2 \mathrm{D}$ and $3 \mathrm{D}$. In $2 \mathrm{D}$, we look for solutions of the form

$$
u(t, x, y) \approx \sum_{k=-\frac{N_{x}}{2}}^{\frac{N_{x}}{2}}, \sum_{l=-\frac{N_{y}}{2}}^{\frac{N_{y}}{2}} \hat{u}_{k, l}(t) e^{i(k x+l y)}, \quad(x, y) \in[0,2 \pi]^{2},
$$

with $N_{x}$ and $N_{y}$ points in the $x$ - and $y$-directions, and appropriate rescaling for different domains. The unknowns, at each time $t$, are the $N_{x} N_{y}$ Fourier coefficients $\hat{u}_{k, l}(t)$. In 3D, we look for solutions of the form

$$
u(t, x, y, z) \approx \sum_{k=-\frac{N_{x}}{2}}^{\frac{N_{x}}{2}}, \sum_{l=-\frac{N_{y}}{2}}^{\frac{N_{y}}{2}} \sum_{m=-\frac{N_{z}}{2}}^{\frac{N_{z}}{2}} \hat{u}_{k, l, m}(t) e^{i(k x+l y+m z)},(x, y, z) \in[0,2 \pi]^{3},
$$

with $N_{x} N_{y} N_{z}$ Fourier coefficients $\hat{u}_{k, l, m}(t)$ at each time $t$. As in $1 \mathrm{D}$, the primes on the summation signs in (3.20)-(3.21) signify that the extreme terms are halved.

To construct differentiation matrices in $2 \mathrm{D}$ and $3 \mathrm{D}$, we use Kronecker products and the $1 \mathrm{D}$ Fourier differentiation matrices. For example, the Laplacian operator in 2D,

$$
\mathcal{L} u=\Delta u=u_{x x}+u_{y y},
$$

is discretized by the $N_{x} N_{y} \times N_{x} N_{y}$ matrix

$$
\mathbf{L}=\mathbf{I}_{N_{y}} \otimes \mathbf{D}_{N_{x}}^{(2)}+\mathbf{D}_{N_{y}}^{(2)} \otimes \mathbf{I}_{N_{x}}
$$

where $\mathbf{I}_{N}$ denotes the identity matrix of size $N$.

Ginzburg-Landau. The (complex) Ginzburg-Landau equation,

$$
u_{t}=(1+i A) \Delta u+u-(1+i B) u|u|^{2},
$$

was first derived in 2D by Stewartson and Stuart in 1971 to study nonlinear instabilities in plane Poiseuille flow [48, using concepts from Ginzburg-Landau theory for superconductivity. The function $u$ is the amplitude of a nonlinear perturbation wave for values of the Reynolds number close to the critical value, above which perturbations may grow. Equation (3.24) admits solutions known as frozen states which correspond to quasi-frozen spiral defects surrounded by shock lines. In this regime, $|u|$ is stationary in time. We take $A=0$ and $B=1.5$,

$$
u(0, x, y)=e^{-0.1\left[(x-50)^{2}+(y-50)^{2}\right]}, \quad(x, y) \in[0,100]^{2},
$$

and

$$
u(0, x, y, z)=e^{-0.1\left[(x-50)^{2}+(y-50)^{2}+(z-50)^{2}\right]}, \quad(x, y, z) \in[0,100]^{3},
$$

and solve up to $t=10$ in both $2 \mathrm{D}$ and $3 \mathrm{D}$. These two initial conditions generate spiral waves. Figure 1.1 shows an example of a $2 \mathrm{D}$ frozen state solution at $t=30$, obtained with an initial condition of amplitude 0.1 involving random noise on the grid. 
Schnakenberg. The Schnakenberg equations,

$$
\left\{\begin{array}{l}
u_{t}=\epsilon_{u} \Delta u+\gamma\left(a-u+u^{2} v\right), \\
v_{t}=\epsilon_{v} \Delta v+\gamma\left(b-u^{2} v\right)
\end{array}\right.
$$

are reaction-diffusion equations derived by Schnakenberg in 1979 to study limit cycle behaviours of two-component chemical reactions [46]. The system (3.27) models the chemical reaction $2 U+V \rightarrow$ $3 U ; U \rightleftharpoons A ; B \rightarrow V ; u$ and $v$ are the concentrations of $U$ and $V$, and $A$ and $B$ are another two chemical species, assumed to be maintained at constant concentrations $a$ and $b$. We take $\epsilon_{u}=1$, $\epsilon_{v}=10, \gamma=3, a=0.1$ and $b=0.9$. The initial conditions are

$$
\begin{aligned}
& u(0, x, y)=1-e^{-2\left[(x-G / 2.15)^{2}+(y-G / 2.15)^{2}\right]} \\
& v(0, x, y)=\frac{0.9}{(0.1+0.9)^{2}}+e^{-2\left[(x-G / 2)^{2}+2(y-G / 2)^{2}\right]}
\end{aligned}
$$

with $(x, y) \in[0, G]^{2}$ and $G=30$ in $2 \mathrm{D}$, and

$$
\begin{aligned}
& u(0, x, y, z)=1-e^{-2\left[(x-G / 2.15)^{2}+(y-G / 2.15)^{2}+(z-G / 2.15)^{2}\right]}, \\
& v(0, x, y, z)=\frac{0.9}{(0.1+0.9)^{2}}+e^{-2\left[(x-G / 2)^{2}+2(y-G / 2)^{2}+2(z-G / 2)^{2}\right]},
\end{aligned}
$$

with $(x, y, z) \in[0, G]^{3}$ and $G=30$ in $3 \mathrm{D}$. We solve up to $t=20$ in both $2 \mathrm{D}$ and $3 \mathrm{D}$. Note that these initial conditions are small perturbations from the constant solution $(u, v)=\left(a+b, b /(a+b)^{2}\right)$. They lead to a set of spots at around $t=500$ in 2D and $t=300$ in 3D. Figure 1.1 shows the 2D solution at $t=500$.

Swift-Hohenberg. The Swift-Hohenberg equation,

$$
u_{t}=r u-(1+\Delta)^{2} u+g u^{2}-u^{3},
$$

was first derived in 2D by Swift and Hohenberg in 1977 to study thermal fluctuations on a fluid near the Rayleigh-Bénard convective instability [50. In 2D, the function $u$ is the temperature field in a plane horizontal layer of fluid heated from below. Equation (3.30) is another example of a PDE that exhibits pattern formation, including stripes, spots and spirals. We take $r=0.1, g=1$,

$$
u(0, x, y)=\frac{1}{4}\left(\sin \left(\frac{\pi x}{10}\right)+\sin \left(\frac{\pi y}{10}\right)+\sin \left(\frac{\pi x}{2}\right) \sin \left(\frac{\pi y}{2}\right)\right)
$$

with $(x, y) \in[0,20]^{2}$ and

$$
\begin{gathered}
u(0, x, y, z)=\frac{1}{4}\left(\sin \left(\frac{\pi x}{10}\right)+\sin \left(\frac{\pi y}{10}\right)+\sin \left(\frac{\pi z}{10}\right)+\sin \left(\frac{\pi x}{2}\right) \sin \left(\frac{\pi y}{2}\right)\right. \\
\left.+\sin \left(\frac{\pi x}{2}\right) \sin \left(\frac{\pi z}{2}\right)+\sin \left(\frac{\pi y}{2}\right) \sin \left(\frac{\pi z}{2}\right)\right),
\end{gathered}
$$

with $(x, y, z) \in[0,20]^{3}$, and solve up to $t=20$ in both $2 \mathrm{D}$ and $3 \mathrm{D}$. Both of these two initial conditions lead to a set of spots. Figure 1.1 shows the $2 \mathrm{D}$ solution at $t=1000$ obtained with $r=0.1, g=0$ and an initial condition of amplitude 0.1 involving random noise on the grid. This solution corresponds to the so-called convection rolls. 

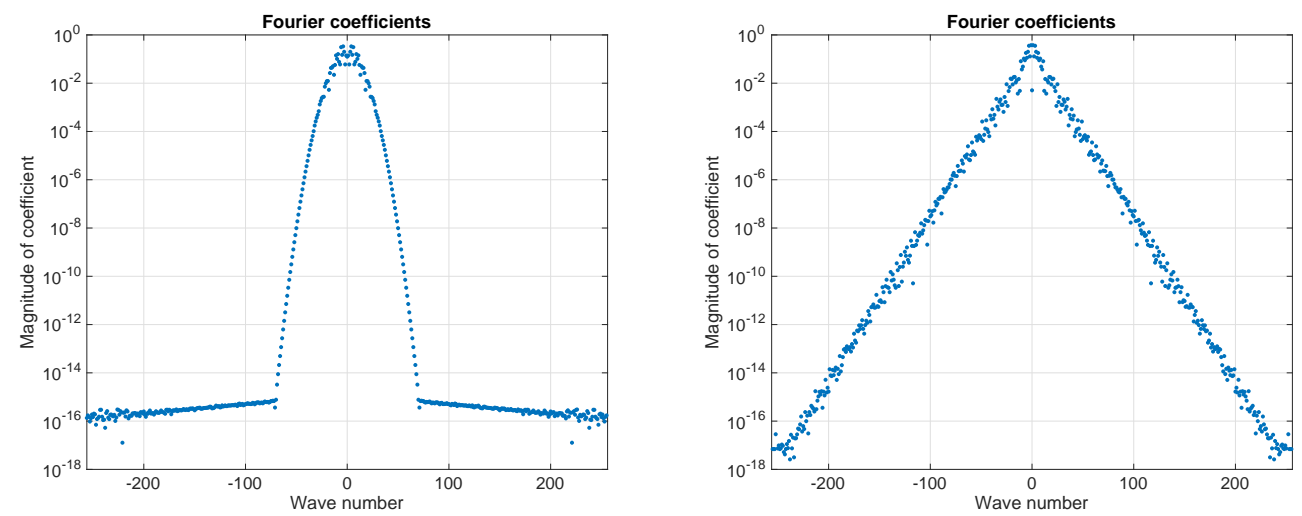

Fig. 4.1: Fourier coefficients of the initial condition (3.3) of the Allen-Cahn equation (left) and of the solution at $t=60$ computed with time-step $h=10^{-2}$ (right). With $N=512$ grid points, the Fourier coefficients decay to about $10^{-16}$. In our computations for the Allen-Cahn equation, the smallest error due to the time discretization is equal to about $10^{-12} \gg 10^{-16}$.

\section{Numerical comparisons.}

4.1. Methodology. To compare exponential integrators, we follow the methodology of [26], though the experiments described here are far more extensive. We solve a given PDE up to $t=T$ for various time-steps $h$ and a fixed number of grid points. We estimate the "exact" solution $u^{e x}(t=T, X)$ by using a "very small" time-step (half the smallest time-step $h$ ) and the PECEC736 scheme (one of the two seventh-order accurate schemes in Table 2.2). We then measure the relative $L^{2}$-error $E$ at $t=T$ between the computed solution $u(t=T, X)$ and $u^{e x}(t=T, X)$, i.e.,

$$
E=\frac{\left\|u(t=T, X)-u^{e x}(t=T, X)\right\|_{2}}{\left\|u^{e x}(t=T, X)\right\|_{2}} .
$$

For both $u$ and $u^{e x}$ we use $N=512$ grid points in $1 \mathrm{D}, N_{x}=N_{y}=128$ grid points in $2 \mathrm{D}$ and $N_{x}=N_{y}=N_{z}=128$ grid points in 3D. (With these grid sizes, the error due to the spatial discretization is small compared to the error due to the time discretization; see Figure 4.1]) For the contour integrals, we use $M=64$ points in $1 \mathrm{D}$ and $M=32$ points in $2 \mathrm{D}$ and $3 \mathrm{D}$. We plot (4.1) against relative time-steps $h / T$ and computer times on a pair of graphs 5 The former gives a measure of the accuracy of the exponential integrator for various time-steps or, equivalently, for various number of integration steps. (If the relative time-step is $10^{-3}$, it means that the integrator performed $10^{3}$ steps to reach $t=T$.) However, it is possible that each step is more costly, so it is the latter that ultimately matters. We compare different families on different pairs of graphs with curves for ETDRK4 included on all plots as a baseline. We have tested every integrator on every PDE, but we shall only show graphs that correspond to the characteristic behaviour of a family of integrators, or highlight notable features such as instability or particularly good/bad performance. The rest of the graphs can be found in the Ph.D. thesis of the first author [38].

\footnotetext{
${ }^{5}$ The precomputation of the coefficients of the exponential integrators and the starting phase of multistep methods are not included in the computing time. Timings were done on a $2.8 \mathrm{GHz}$ Intel i7 machine with $16 \mathrm{~GB}$ of RAM.
} 
4.2. Starting multistep schemes. To start a multistep scheme with $q$ steps, one needs $q$ values: the initial condition $\hat{u}^{0}$ and $q-1$ extra values $\hat{u}^{1}, \ldots, \hat{u}^{q-1}$. It is suggested in [8] to use the following strategy: first, compute an approximation of $\hat{U}=\left(\hat{u}^{1}, \ldots, \hat{u}^{q-1}\right)^{T}$ using a low-order exponential integrator (e.g., ETDRK2, the second-order version of ETDRK4, also introduced by Cox and Matthews in [12]), and then, use a fixed point iteration to refine this approximation. The fixed point iteration is applied to the following system of nonlinear equations,

$$
\hat{u}^{j}=e^{j h \mathbf{L}} \hat{u}^{0}+h \sum_{l=0}^{q-1} \gamma_{l}(j, h \mathbf{L}) \Delta^{l} \mathbf{N}\left(\hat{u}^{0}\right), \quad 1 \leq j \leq q-1,
$$

where $\Delta^{l}$ is the forward difference operator,

$$
\Delta^{0} \mathbf{N}\left(\hat{u}^{0}\right)=\hat{u}^{0}, \quad \Delta^{l} \mathbf{N}\left(\hat{u}^{0}\right)=\sum_{i=0}^{l}(-1)^{i}\left(\begin{array}{l}
l \\
i
\end{array}\right) \mathbf{N}\left(\hat{u}^{l-i}\right), \quad l \geq 1,
$$

and the $\gamma$-functions are defined by the recurrence relation,

$$
\begin{aligned}
& \gamma_{0}(k, z)=\frac{e^{k z}-1}{z}, \\
& \gamma_{j}(k, z)=\frac{\left(\sum_{m=1}^{j} \frac{(-1)^{m-1}}{m} \gamma_{j-m}(k, z)\right)-\left(\begin{array}{c}
k \\
j
\end{array}\right)}{z}, \quad 1 \leq j \leq k, \\
& \gamma_{j}(k, z)=\frac{\left(\sum_{m=1}^{j} \frac{(-1)^{m-1}}{m} \gamma_{j-m}(k, z)\right)}{z}, \quad j>k .
\end{aligned}
$$

Note that, like the $\varphi$-functions, the $\gamma$-functions can be evaluated by contour integrals and satisfy the symmetry property $\gamma(\bar{z})=\bar{\gamma}(z)$ for all $z \in \mathbb{C}$. Let us write (4.2) as $\hat{U}=F(\hat{U})$. The fixed point iteration is then given by

$$
\hat{U}_{[n+1]}=F\left(\hat{U}_{[n]}\right),
$$

where $\hat{U}_{[n]}=\left(\hat{u}_{[n]}^{1}, \ldots, \hat{u}_{[n]}^{q-1}\right)^{T}$ denotes the approximation obtained after $n$ iterations $\left(\hat{U}_{[0]}\right.$ corresponding to the approximation given by ETDRK2). The fixed point iteration (4.5) is carried out until the norm of the difference between two successive iterations is of the order of $h^{q}$.

4.3. Results. We now report on the results of our numerical testing and present plots showing the typical behaviours and notable features we see from each set of methods.

Figure 4.2 shows results for the ETD Adams-Bashforth methods for the Kuramoto-Sivashinsky equation (1D). These formulas are often unstable for large time-steps but can be competitive at high accuracies. For the Cahn-Hilliard and KdV equations (Figures C.3 and C.5 in [38]), we could not get them to work at all with the spatial discretization that we used.

Figure 4.3 shows results for the ETD Runge-Kutta methods for the 1D Cahn-Hilliard, 1D KdV and 2D Swift-Hohenberg equations. These formulas have good stability properties. The fourthorder methods have similar performance to ETDRK4. The fifth-order EXPRK5S8 integrator is 

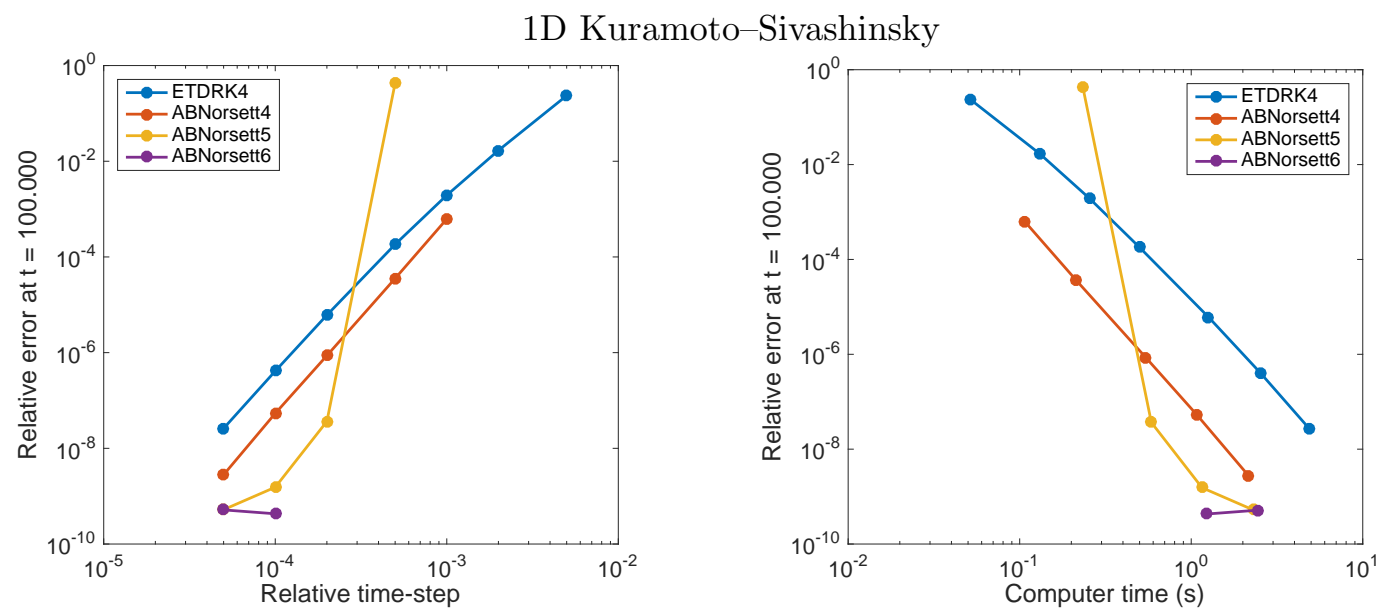

Fig. 4.2: Accuracy versus time-step and computer time for the ETD Adams-Bashforth methods for the $1 D$ Kuramoto-Sivashinsky equation. The order 5 and 6 methods are more efficient than ETDRK 4 at high accuracies, but often unstable at lower accuracies, as reflected in dots missing from the curves.

impressively efficient in $1 \mathrm{D}$, but exhibits instability for the Cahn-Hilliard and KdV equations. In $2 \mathrm{D}$ and $3 \mathrm{D}$, it is more accurate than the fourth-order methods for the six PDEs we have considered, and more efficient for the Ginzburg-Landau equation (Figures C.11 and C.17 in [38). However, for the Schnakenberg (Figures C.13 and C.19 in [38]) and Swift-Hohenberg equations, it is not accurate enough to counterbalance its high cost per time-step.

Figure 4.4 shows results for the Lawson methods for the NLS equation (1D). These formulas are not accurate enough to be competitive. For the ABLawson4 formula, this lack of accuracy is partly compensated by its low computational cost per time-step (it is a purely multistep method).

Figure 4.5 shows results for the generalised Lawson methods for the 1D Kuramoto-Sivashinsky and 2D Ginzburg-Landau equations. In 1D, these formulas suffer from instabilities for most PDEs. In $2 \mathrm{D}$ and $3 \mathrm{D}$, the GenLawson41 formula has virtually identical performance to ETDRK4, the GenLawson42 formula is always less efficient than ETDRK4, while the other variants with three to five steps perform well for the Ginzburg-Landau equation but are less efficient for the Schnakenberg and Swift-Hohenberg equations (Figures C.14, C.16, C.20 and C.22 in [38]).

Figure 4.6 shows results for the modified generalised Lawson methods for the 1D KuramotoSivashinsky, 1D KdV and 3D Swift-Hohenberg equations. In 1D, these formulas are much more stable than the generalised Lawson schemes and are quite efficient, but still suffer from instabilities for the $1 \mathrm{D} \mathrm{KdV}$ equation. In $2 \mathrm{D}$ and $3 \mathrm{D}$, we reach the same conclusions as for the generalised Lawson methods: the ModGenLawson41 formula has virtually identical performance to ETDRK4, the ModGenLawson42 formula is always the least efficient and the other variants perform well for some problems but are less efficient for others.

Figure 4.7 shows results for the exponential predictor-corrector methods for the 1D Allen-Cahn, 1D Cahn-Hilliard and 2D Schnakenberg equations. These formulas are particularly efficient in 1D, especially for the Kuramoto-Sivashinsky and NLS equations (Figures C.8 and C.10 in [38]), but 
1D Cahn-Hilliard
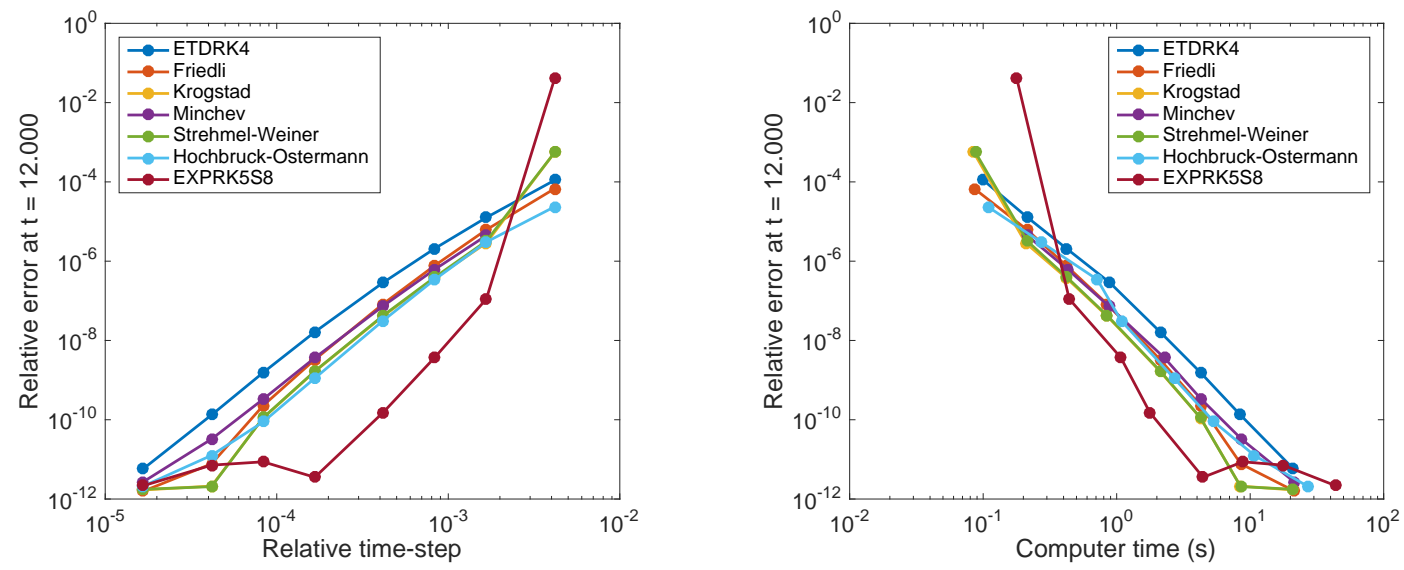

1D Korteweg-de Vries
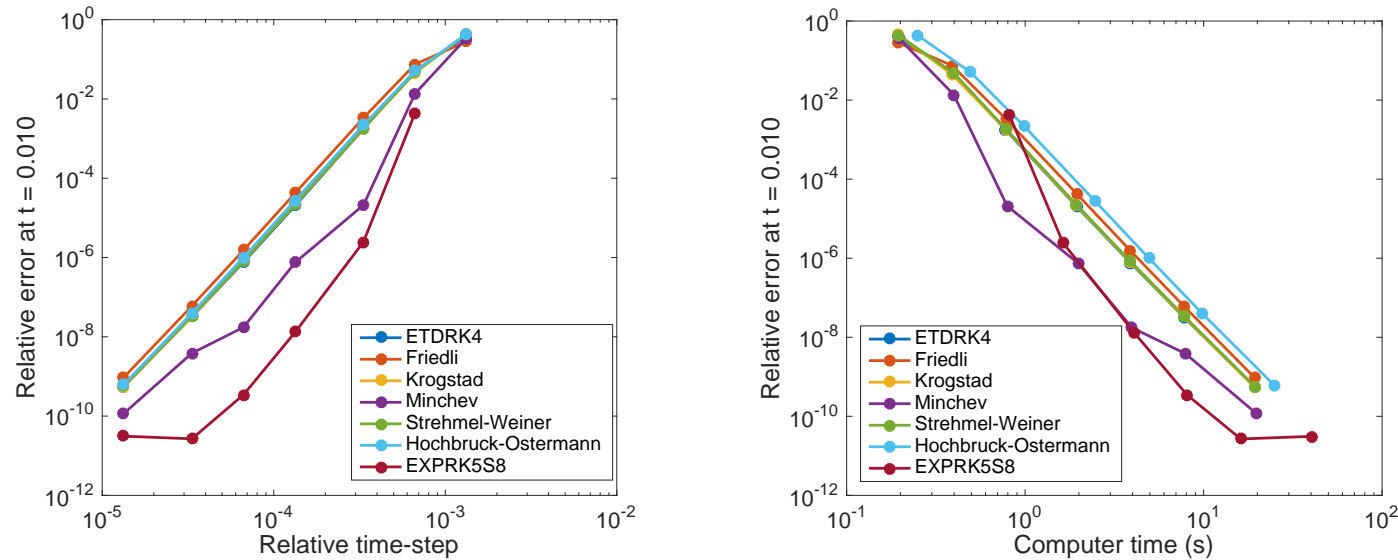

2D Swift-Hohenberg
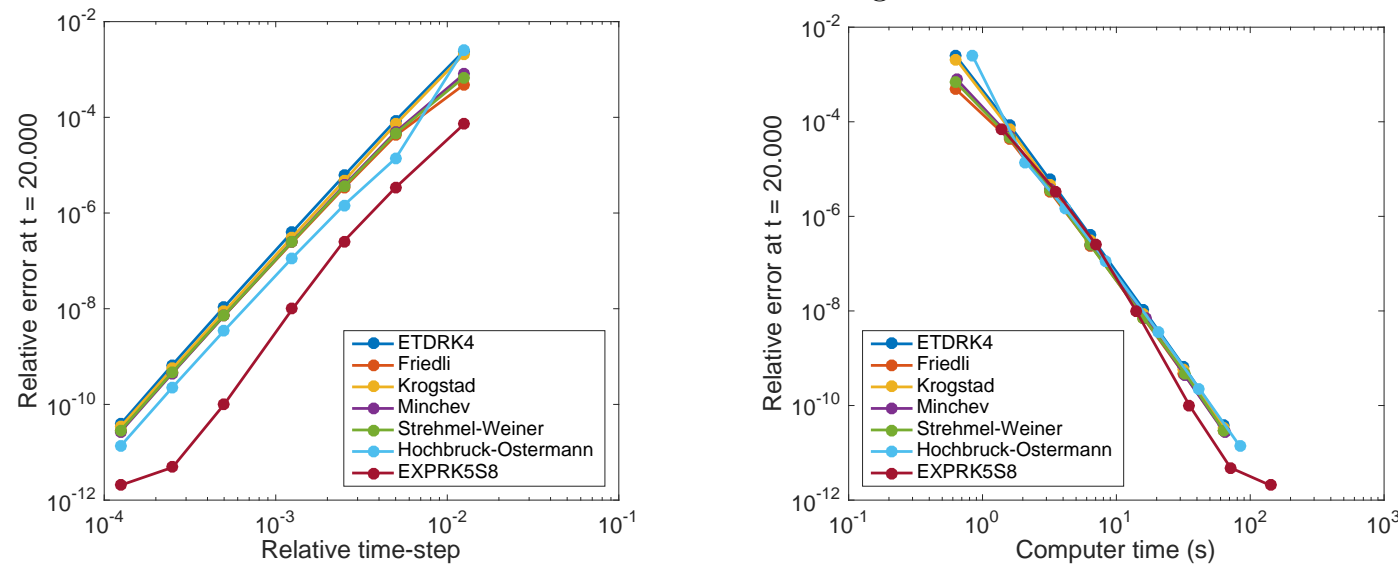

Fig. 4.3: Accuracy versus time-step and computer time for the ETD Runge-Kutta methods for the $1 D$ Cahn-Hilliard (top), $1 D K d V$ (centre) and $2 D$ Swift-Hohenberg (bottom) equations. The EXPRK $5 S 8$ scheme is impressively efficient in $1 D$ but is unstable at low accuracies for the CahnHilliard and $K d V$ equations. In $2 D$ and $3 D$, it does not beat the fourth-order methods for the Schnakenberg (Figures C.13 and C.19 in [38]) and Swift-Hohenberg equations (above for 2D, Figure C.21 in [38] for 3D). 
1D nonlinear Schrödinger
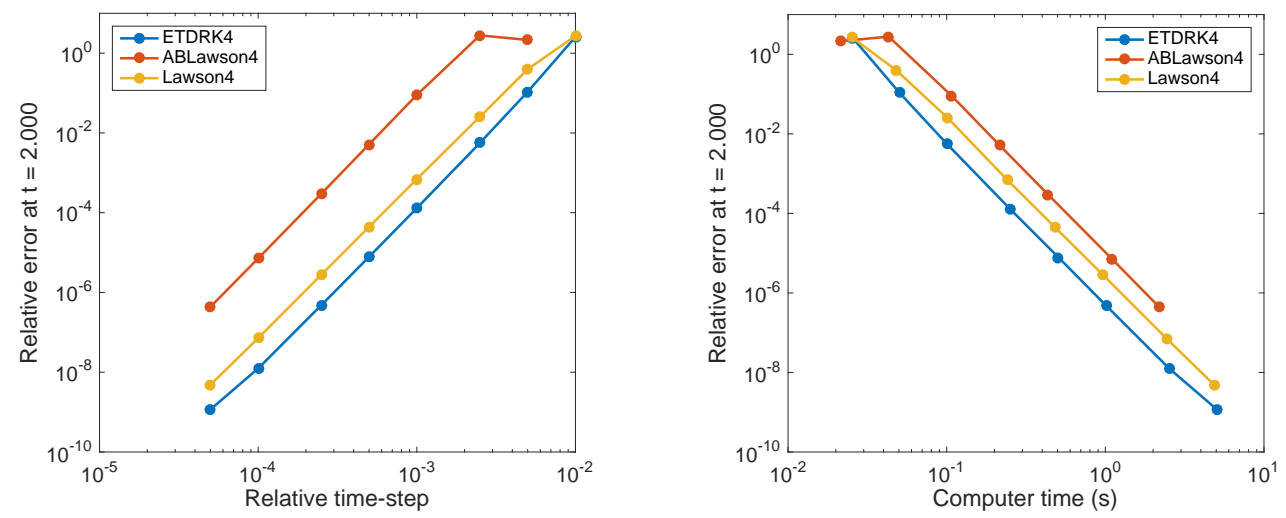

Fig. 4.4: Accuracy versus time-step and computer time for the Lawson methods for the $1 D N L S$ equation. These formulas are too inaccurate to be competitive; the constants involved in the convergence bounds are too great.

do not clearly outperform ETDRK4 for the Allen-Cahn equation. Most of them are unstable at low accuracies for the Cahn-Hilliard equation, especially the higher-order schemes, and some of them are also unstable at low accuracies for the KdV equation (Figure C.6 in [38]). In 2D and 3D, they are more efficient than ETDRK4 for the Ginzburg-Landau equation (Figures C.12 and C.18 in [38]) but most of them have similar performance to it for the other PDEs we have considered. Note that the higher-order schemes with two steps (PEC625 and PEC726) also beat ETDRK4 for the Swift-Hohenberg equations, but these are particularly unstable for the Cahn-Hilliard and KdV equations.

5. Discussion. We have tested 30 exponential integrators on 11 model problems in 1D, 2D and 3D, and have observed considerable differences in stability and efficiency. As expected, the schemes did not exhibit any order reduction (periodic boundary conditions). The main conclusion is that it is difficult to find a method that outperforms ETDRK4 for all the PDEs we have considered.

Our experiments show that the ETD Adams-Bashforth and the generalised Lawson methods are highly unstable while the Lawson methods are not accurate enough. Within the ETD RungeKutta methods, it is hard to do much better than ETDRK4. The fourth-order schemes are quite similar in terms of efficiency and stability. The fifth-order EXPRK5S8 integrator is more efficient than ETDRK4 for most PDEs in 1D, but is unstable at low accuracies for the KdV and CahnHilliard equations. In 2D and 3D, it outperforms ETDRK4 only for the Ginzburg-Landau equation. Since it requires the precomputation of more than twice as many coefficients as ETDRK4, it makes it much more complicated to implement and probably less appealing to general users. The highorder modified generalised Lawson and exponential predictor-corrector methods are competitive stiff solvers for some PDEs, but for others do not outperform ETDRK4 or else suffer from instabilities.

Our numerical experiments were performed using MATLAB and have been embedded within Chebfun. More specifically, the spin, spin2 and spin3 codes implement a Fourier spectral method and exponential integrators to solve PDEs in 1D, 2D and 3D periodic domains. (Note that spin stands for stiff PDE integrator.) These have been one of the most major additions to Chebfun in 

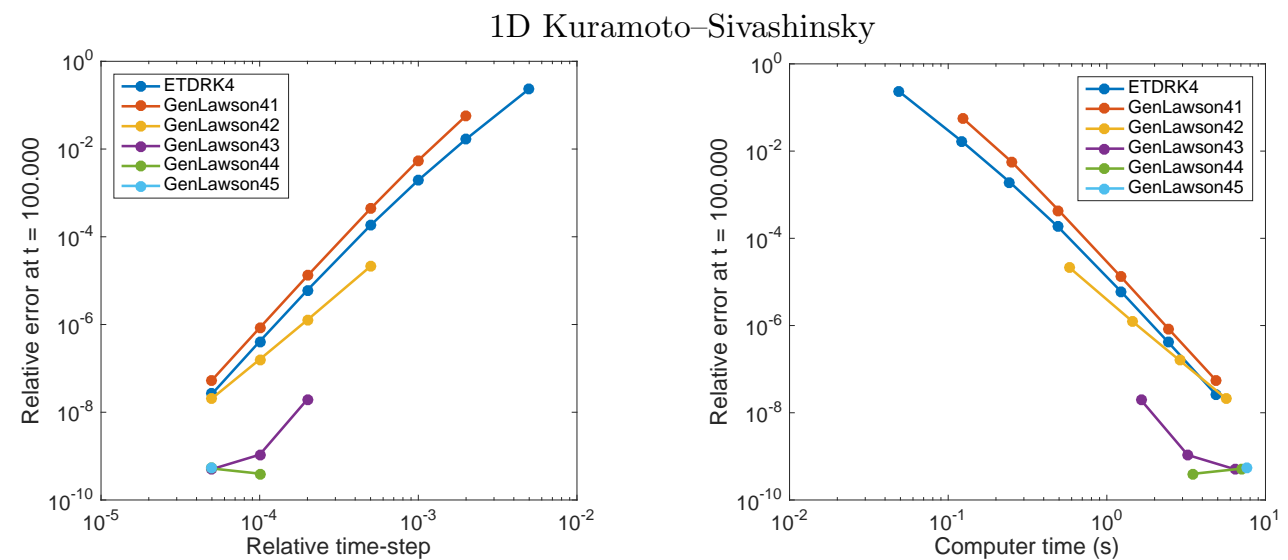

2D Ginzburg-Landau
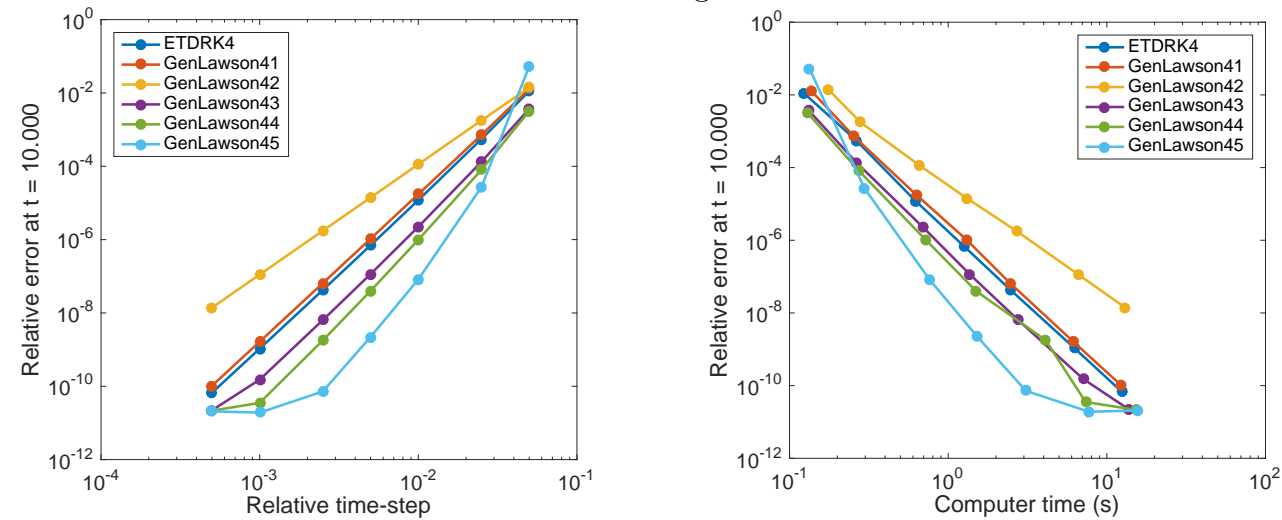

Fig. 4.5: Accuracy versus time-step and computer time for the generalised Lawson methods for the $1 D$ Kuramoto-Sivashinsky (top) and 2D Ginzburg-Landau (bottom) equations. These methods are highly unstable for most PDEs in $1 D$. In $2 D$ and $3 D$, the GenLawson 41 formula has virtually identical performance to ETDRK 4 while the other variants with three to five steps perform well for some problems (e.g., 2D Ginzburg-Landau equation above) but are less efficient for others (e.g., Figure C.14 in [38]).

recent years from a user point of view. The simplest way to see spin in action is to type simply spin('ks') (for the Kuramoto-Sivashinsky equation) or spin2('gl2') (for the 2D GinzburgLandau equation) to invoke an example computation. It is also possible to define your own PDE using the spinop class. To produce the graphs of Section 4.3, we have used the spincomp code.

Acknowledgements. This paper is dedicated to Nick Trefethen for his inspirational contributions to the field of numerical analysis. 
1D Kuramoto-Sivashinsky
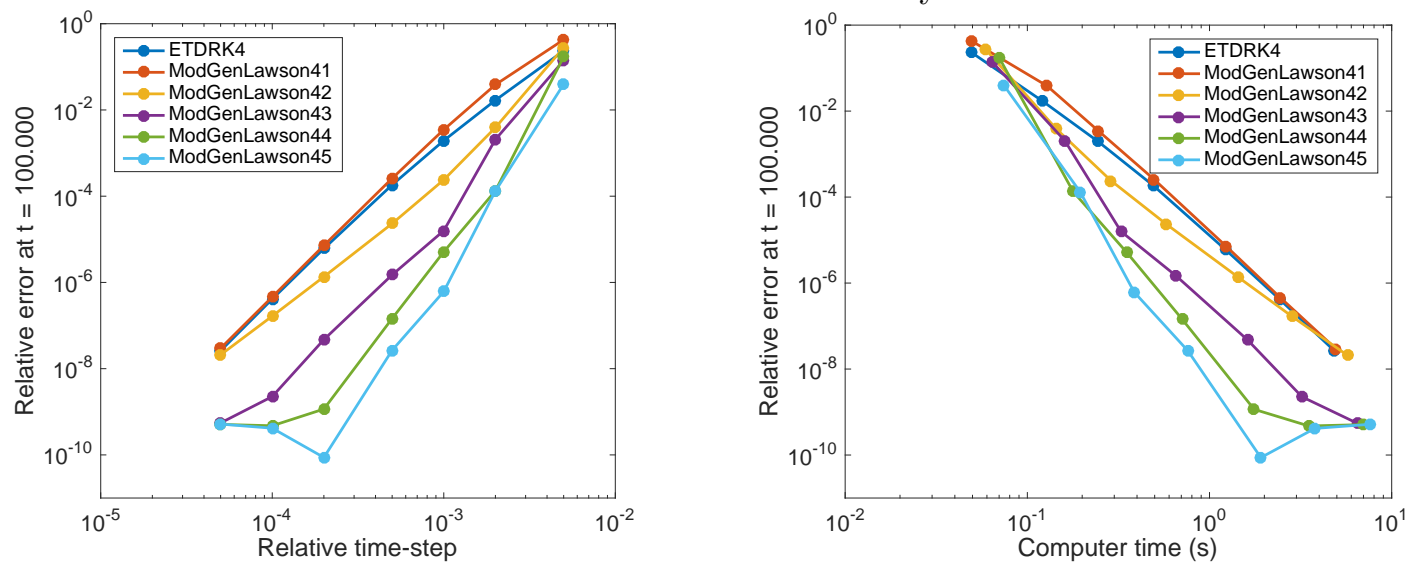

1D Korteweg-de Vries
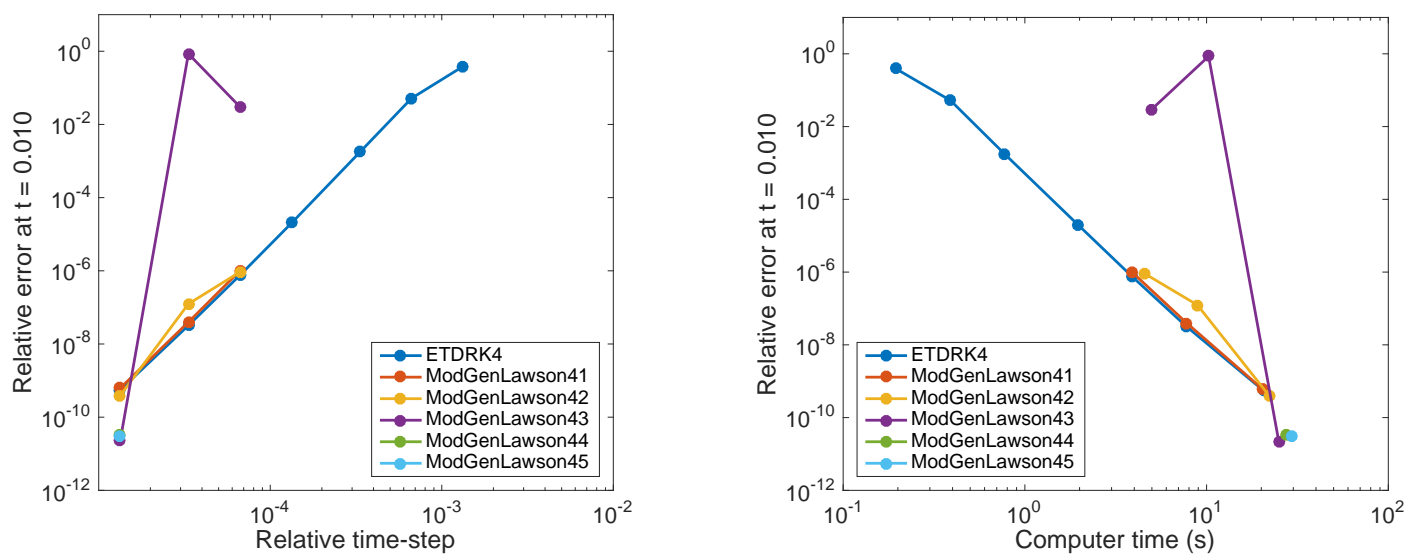

3D Swift-Hohenberg
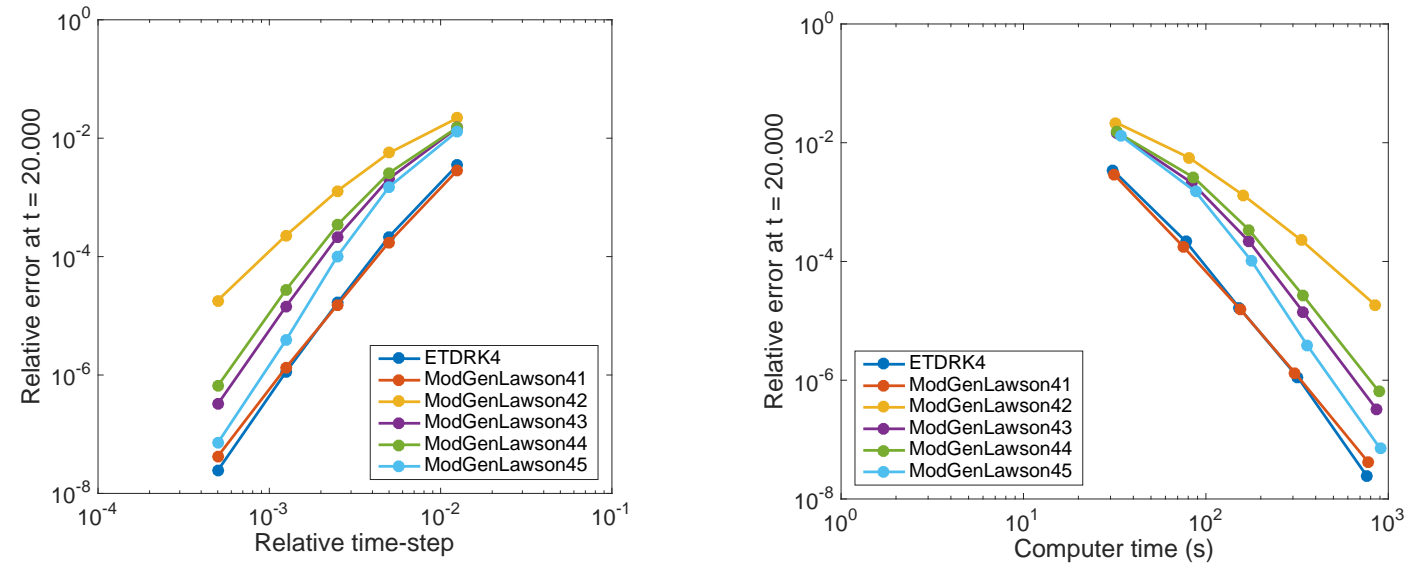

Fig. 4.6: Accuracy versus time-step and computer time for the modified generalised Lawson methods for the $1 D$ Kuramoto-Sivashinsky (top), $1 D \mathrm{KdV}$ (centre) and $3 D$ Swift-Hohenberg (bottom) equations. In $1 D$, these methods are much more stable than the generalised Lawson methods but are still highly unstable for the KdV equation. In $2 D$ and $3 D$, they are very similar to the generalised Lawson methods, i.e., they perform well for some problems (e.g., Figure C.18 in [38]) but are less efficient for others (e.g., 3D Swift-Hohenberg equation above). 
1D Allen-Cahn
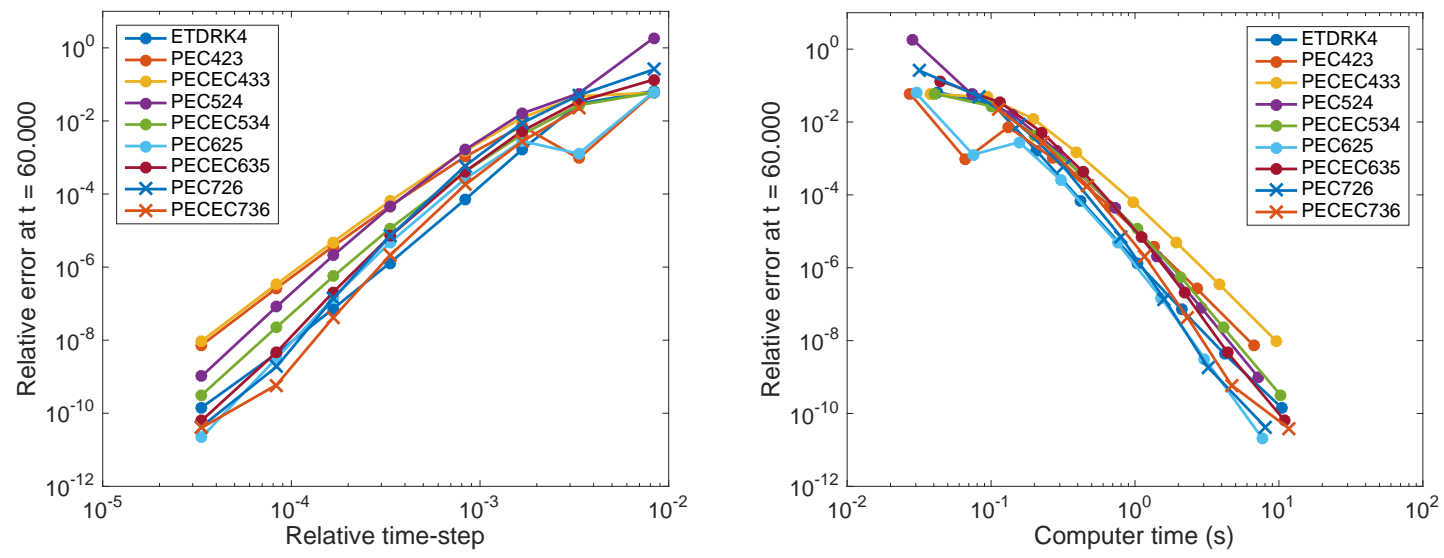

1D Cahn-Hilliard
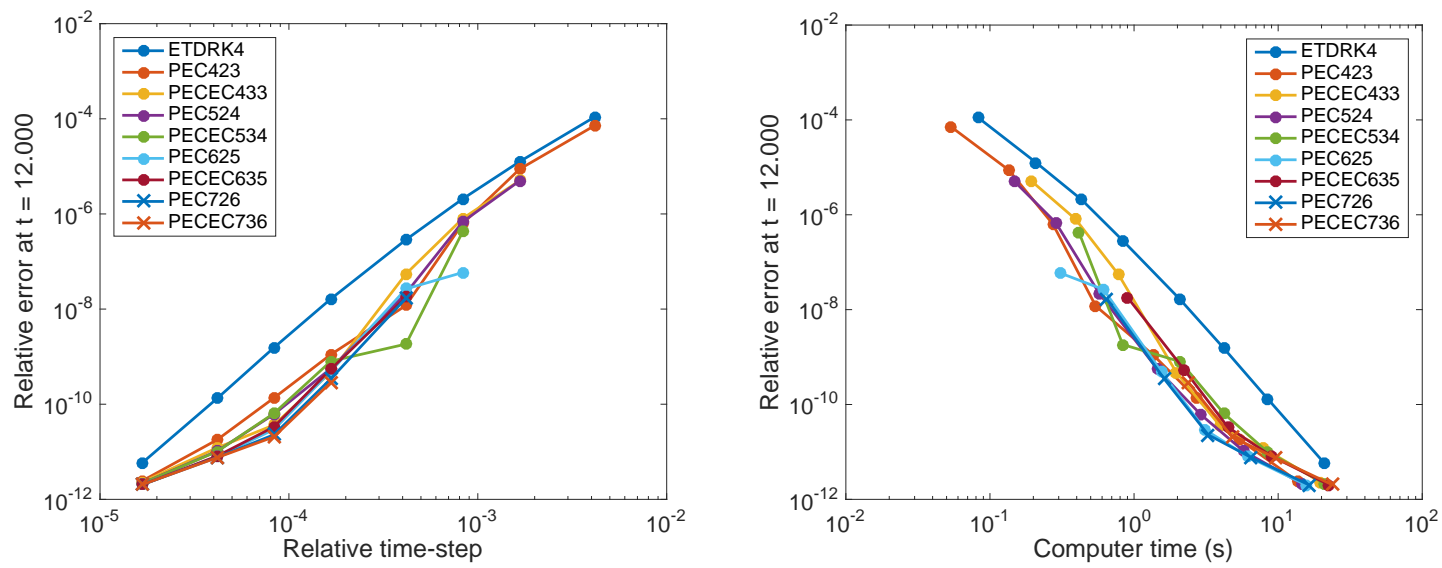

2D Schnakenberg
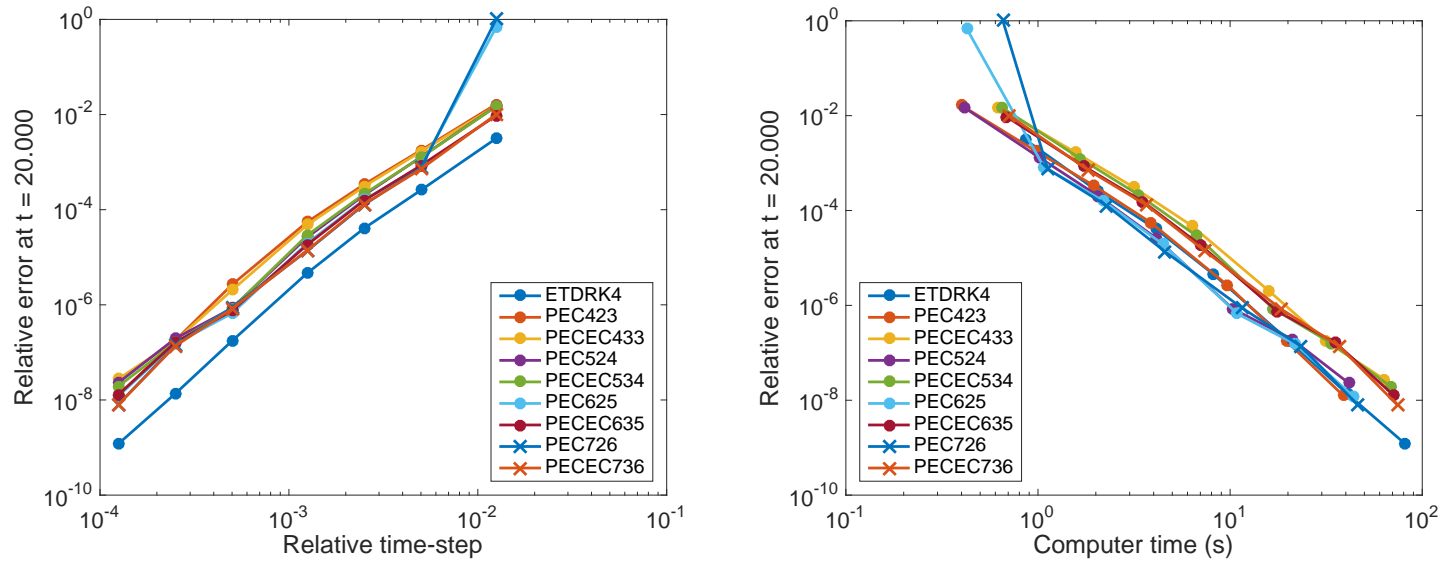

Fig. 4.7: Accuracy versus time-step and computer time for the predictor-corrector methods for the $1 D$ Allen-Cahn (top), $1 D$ Cahn-Hilliard (centre) and $2 D$ Schnakenberg equations (bottom). In $1 D$, these schemes are efficient, but do not clearly outperform ETDRK 4 for the Allen-Cahn equation and are unstable at low accuracies for the Cahn-Hilliard equation. In $2 D$ and $3 D$, they beat ETDRK 4 for the Ginzburg-Landau equation (Figures C.12 and C.18 in [38]) but have similar performance for the other equations (e.g., 2D Schnakenberg equations above). 
[1] A. H. Al-Mohy And N. J. Higham, Computing the action of the matrix exponential, with an application to exponential integrators, SIAM J. Sci. Comput., 33 (2011), pp. 488-511.

[2] S. M. Allen And J. W. Cahn, A microscopic theory for antiphase boundary motion and its application to antiphase domain coarsening, Acta Metall., 27 (1979), pp. 1085-1095.

[3] U. M. Ascher, S. J. Ruuth, and B. T. R. Wetton, Implicit-explicit methods for time-dependent partial differential equations, SIAM J. Numer. Anal., 32 (1995), pp. 797-823.

[4] H. Berland, B. Skaflestad, and W. M. Wright, EXPINT-A MATlaB package for exponential integrators, ACM Trans. Math. Softw. (TOMS), 33 (2007), pp. 4:1-4:17.

[5] G. Beyklin, J. M. Keiser, And L. Vozovoi, A new class of time discretization schemes for the solution of nonlinear PDEs, J. Comput. Phys., 147 (1998), pp. 362-387.

[6] N. J. Bootland, Exponential integrators for stiff PDEs, Master's thesis, University of Oxford, 2014.

[7] J. W. Cahn and J. E. Hilliard, Free energy of a nonuniform system. I. Interfacial free energy, The Journal of Chemical Physics, 28 (1958), pp. 258-267.

[8] M. P. Calvo and C. Palencia, A class of multistep exponential integrators for semilinear problems, Numer. Math., 102 (2006), pp. 367-381.

[9] B. Cano and A. Gonzáles-Pachón, Exponential time integration of solitary waves of cubic Schrödinger equation, Appl. Numer. Math., 91 (2015), pp. 26-45.

[10] - Projected explicit Lawson methods for the integration of Schrödinger equation, Numer. Methods Partial Differential Eq., 31 (2015), pp. 78-104.

[11] J. Certaine, The solution of ordinary differential equations with large time constants, in Mathematical methods for digital computers, A. Ralston and H. S. Wilf, eds., Wiley, New York, 1960, pp. 128-132.

[12] S. M. Cox And P. C. Matthews, Exponential time differencing for stiff systems, J. Comput. Phys., 176 (2002), pp. 430-455.

[13] T. A. Driscoll, N. Hale, and L. N. Trefethen, eds., Chebfun Guide, Pafnuty Publications, Oxford, 2014; see also www. chebfun.org

[14] B. L. Ehle And J. D. Lawson, Generalized Runge-Kutta processes for stiff initial-value problems, J. Inst. Maths. Appl., 16 (1975), pp. 11-21.

[15] A. FriedLI, Verallgemeinerte Runge-Kutta Verfahren zur Lösung steifer Differentialgleichungssysteme, in Numerical Treatment of Differential Equations, R. Burlirsch, R. Grigorieff, and J. Schröder, eds., Springer, Berlin, 1978, pp. 35-50.

[16] F. Garcia, L. Bonaventura, M. Net, and J. Sánchez, Exponential versus IMEX high-order time integrators for thermal convection in rotating spherical shells, J. Comput. Phys., 264 (2014), pp. 41-54.

[17] E. Hairer, G. Bader, And C. Lubich, On the stability of semi-implicit methods for ordinary differential equations, BIT, 22 (1982), pp. 211-232.

[18] E. Hairer and G. Wanner, Solving Ordinary Differential Equations II: Stiff and Differential-Algebraic Problems, Springer, New York, 1991.

[19] J. Hersch, Contribution à la méthode des équations aux différences, Z. Angew. Math. und Phys., 9 (1958), pp. 129-180.

[20] M. Hochbruck, C. Lubich, and H. Selhofer, Exponential integrators for large systems of differential equations, SIAM J. Sci. Comput., 19 (1998), pp. 1552-1574.

[21] M. Hochbruck and A. Ostermann, Explicit exponential Runge-Kutta methods for semilinear parabolic problems, SIAM J. Numer. Anal., 43 (2005), pp. 1069-1090.

[22] - Exponential integrators, Acta Numer., 19 (2010), pp. 209-286.

[23] —, Exponential multistep methods of Adams-type, BIT, 51 (2011), pp. 889-908.

[24] M. Hochbruck, A. Ostermann, and J. Schweitzer, Exponential Rosenbrock-type methods, SIAM J. Numer. Anal., 47 (2009), pp. 786-803.

[25] A.-K. Kassam, Solving reaction-diffusion equations 10 times faster, Tech. Rep. 1192, Numerical Analysis Group, University of Oxford, 2003.

[26] A.-K. Kassam and L. N. Trefethen, Fourth-order time-stepping for stiff PDEs, SIAM J. Sci. Comput., 26 (2005), pp. 1214-1233.

[27] C. KLeIN, Fourth order time-stepping for low dispersion Korteweg-de Vries and nonlinear Schrödinger equations, Electron. Trans. Numer. Anal., 29 (2008), pp. 116-135.

[28] C. Klein and K. Roidot, Fourth order time-stepping for Kadomtsev-Petviashvili and Davey-Stewartson equations, SIAM J. Sci. Comput., 33 (2011), pp. 3333-3356.

[29] D. Korteweg and G. De VRies, On the change of form of long waves advancing in a rectangular canal, and on a new type of long stationary waves, Philos. Mag. (Ser. 5), 39 (1895), pp. 422-443.

[30] S. Krogstad, Generalized integrating factor methods for stiff PDEs, J. Comput. Phys., 203 (2005), pp. 72-88.

[31] Y. Kuramoto And T. Tsuzuki, Persistent propagation of concentration waves in dissipative media far from 
thermal equilibrium, Progress of Theoretical Physics, 55 (1976), pp. 356-369.

[32] J. Lawson, Generalized Runge-Kutta processes for stable systems with large Lipschitz constants, SIAM J. Numer. Anal., 4 (1967), pp. 372-380.

[33] J. Loffeld and M. Tokman, Comparative performance of exponential, implicit, and explicit integrators for stiff systems of ODEs, J. Comput. Appl. Math., 241 (2013), pp. 45-67.

[34] V. T. Luan and A. Ostermann, Explicit exponential Runge-Kutta methods of high order for parabolic problems, J. Comput. Appl. Math., 256 (2014), pp. 168-179.

[35] - Exponential Rosenbrock methods of order five-construction, analysis and numerical comparisons, J. Comput. Appl. Math., 255 (2014), pp. 417-431.

[36] B. V. Minchev, Exponential integrators for semilinear problems, PhD thesis, University of Bergen, 2004.

[37] B. V. Minchev and W. M. Wright, A review of exponential integrators for first order semi-linear problems, Tech. Rep. 2/2005, Norwegian University of Science and Technology, 2005.

[38] H. Montanelli, Numerical algorithms for differential equations with periodicity, PhD thesis, University of Oxford, 2017.

[39] S. P. NøRsett, An A-stable modification of the Adams-Bashforth methods, in Conference on the numerical solution of differential equations (Dundee, 1969), Springer, Berlin, 1969, pp. 214-219.

[40] A. Ostermann, M. Thalhammer, and W. M. Wright, A class of explicit exponential general linear methods, BIT, 46 (2006), pp. 409-431.

[41] D. A. Pope, An exponential method of numerical integration of ordinary differential equations, Communications of the ACM, 6 (1963), pp. 491-493.

[42] G. Rainwater and M. Tokman, A new class of split exponential propagation iterative methods of RungeKutta type (sEPIRK) for semilinear systems of ODEs, J. Comput. Phys., 269 (2014), pp. 40-60.

[43] - A new approach to constructing efficient stiffly accurate EPIRK methods, J. Comput. Phys., 323 (2016), pp. 283-309.

[44] H. H. Rosenbrock, Some general implicit processes for the numerical solution of differential equations, Computer, 5 (1963), pp. 329-330.

[45] T. Schmelzer and L. N. Trefethen, Evaluating matrix functions for exponential integrators via Carathéodory-Fejér approximation and contour integrals, Electron. Trans. Numer. Anal., 29 (2007), pp. 118.

[46] J. Schnakenberg, Simple chemical reaction systems with limit cycle behaviour, J. Theor. Biol., 81 (1979), pp. 389-400.

[47] G. I. Sivashinsky, Nonlinear analysis of hydrodymanic instability in laminar flames-I. Derivation of basic equations, Acta Astronaut., 4 (1977), pp. 1177-1206.

[48] K. Stewartson and J. T. Stuart, A non-linear instability theory for a wave system in plane Poiseuille flow, J. Fluid Mech., 48 (1971), pp. 529-545.

[49] K. Strehmel and R. Weiner, Behandlung steifer Anfangswertprobleme gewöhnlicher Differentialgleichungen mit adaptiven Runge-Kutta-Methoden, Computing, 29 (1982), pp. 153-165.

[50] J. Swift And P. C. Hohenberg, Hydrodynamic fluctuations at the convective instability, Phys. Rev. A, 15 (1977), pp. 319-328.

[51] M. ToKman, Efficient integration of large stiff systems of ODEs with exponential propagation iterative (EPI) methods, J. Comput. Phys., 213 (2006), pp. 748-776.

[52] — A new class of exponential propagation iterative methods of Runge-Kutta type (EPIRK), J. Comput. Phys., 230 (2011), pp. 8762-8778.

[53] M. Tokman and J. Loffeld, Efficient design of exponential-Krylov integrators for large scale computing, Procedia Comp. Sci., 1 (2012), pp. 229-237.

[54] M. Tokman, J. Loffeld, and P. Tranquilli, New adaptive exponential propagation iterative methods of Runge-Kutta type, SIAM J. Sci. Comput., 34 (2012), pp. A2650-A2669.

[55] L. N. Trefethen, Spectral Methods in MATLAB, SIAM, Philadelphia, 2000.

[56] L. N. Trefethen and J. A. C. Weideman, The exponentially convergent trapezoidal rule, SIAM Rev., 56 (2014), pp. 385-458.

[57] L. N. Trefethen, J. A. C. Weideman, and T. Schmelzer, Talbot quadratures and rational approximations, BIT, 46 (2006), pp. 653-670.

[58] A. M. Turing, The chemical basis of morphogenesis, Phil. Trans. Roy. Soc. Lon. (Ser. B), 237 (1952), pp. 3772 .

[59] P. J. van Der Houwen, Construction of Integration Formulas for Initial Value Problems, North-Holland Publishing Co., Amsterdam, 1977.

[60] G. B. Wright, M. Javed, H. Montanelli, and L. N. Trefethen, Extension of Chebfun to periodic functions, SIAM J. Sci. Comput., 37 (2015), pp. C554-C573. 